\title{
The Stability and Slow Dynamics of Two-Spike Patterns for a Class of Reaction-Diffusion System
}

\author{
Y. Nec, M. J. Ward * \\ Department of Mathematics, University of British Columbia \\ 1984 Mathematics Road, Vancouver, V6T1Z2, BC, Canada
}

\begin{abstract}
The slow dynamics and linearized stability of a two-spike quasi-equilibrium solution to a general class of reaction-diffusion (RD) system with and without sub-diffusion is analyzed. For both the case of regular and sub-diffusion, the method of matched asymptotic expansions is used to derive an ODE characterizing the spike locations in the absence of any $\mathcal{O}(1)$ time-scale instabilities of the two-spike quasi-equilibrium profile. These fast instabilities result from unstable eigenvalues of a certain nonlocal eigenvalue problem (NLEP) that is derived by linearizing the RD system around the two-spike quasi-equilibrium solution. For a particular sub-class of the reaction kinetics, it is shown that the discrete spectrum of this NLEP is determined by the roots of some simple transcendental equations. From a rigorous analysis of these transcendental equations, explicit sufficient conditions are given to predict the occurrence of either Hopf bifurcations or competition instabilities of the two-spike quasi-equilibrium solution. The theory is illustrated for several specific choices of the reaction kinetics.
\end{abstract}

Keywords and phrases: matched asymptotic expansions, bifurcation, spikes, nonlocal eigenvalue problem, Hopf bifurcation, sub-diffusion

Mathematics Subject Classification: 35Q53, 34B20, 35G31

\section{Introduction}

The main goal of this paper is to characterize analytically the slow dynamics and the stability of localized multi-pulse, or spike-type, solutions to the following class of reaction-diffusion (RD) system on the infinite line:

$$
v_{t}=\varepsilon_{0}^{2} v_{x x}-v+g(u) v^{p}, \quad \tau u_{t}=u_{x x}+\left(u_{b}-u\right)+\varepsilon_{0}^{-1} f(u) v^{r}, \quad-\infty<x<\infty, \quad t>0 .
$$

Here $\varepsilon_{0} \ll 1, u_{b}$ is a constant, $p>1, r>1$, and the properties of $g(u)$ and $f(u)$ are given below. Since (1.1) is posed on the infinite line, without loss of generality the diffusivity of $u$ can be set to unity (as we have done) by a spatial re-scaling. In the limit $\varepsilon_{0} \rightarrow 0$, localized pulses or spikes in the $v$-variable are represented by homoclinic solutions of spatial extent $\mathcal{O}\left(\varepsilon_{0}\right)$ of the stationary problem for $v$, with the interaction between the spikes mediated by the "global" component $u$ that varies on an $\mathcal{O}(1)$ spatial

\footnotetext{
* Corresponding author. E-mail: ward@math.ubc.ca
} 
scale. This asymptotic limit, where only one of the two components of the RD system is localized, is called the semi-strong spike interaction limit (cf. [5]).

Reaction-diffusion systems of the general class (1.1) arise in various applications. In particular, Turing [33] proposed that localized peaks in the concentration of a chemical substance, known as a morphogen, could be responsible for the process of morphogenesis, which describes the development of a complex organism from a single cell. Through the use of a linearized analysis, he showed how stable spatially complex patterns can develop from small perturbations of spatially homogeneous initial data for a coupled system of reaction-diffusion equations. Later, Gierer and Meinhardt [8] (see the more recent book [17]), have demonstrated numerically the existence of stable spatially inhomogeneous equilibrium solutions for activator-inhibitor systems of the type (1.1) with $u_{b}=0$ where the inhibitor $u$ diffuses more slowly than the activator $v$. A class of GM models is $g(u)=u^{-q}, f(u)=u^{-s}, u_{b}=0$, where $q r /(p-1)-(s+1)>0$ (see [12]). Another application of (1.1) is to the study of chemical patterns, involving two reactants in a gel reactor where the reactor is maintained in contact with a reservoir of one of the two chemical species. This leads to the source or feed term $u_{b}$ in (1.1). In this class of substrate-depletion models, a ubiquitous model is the Gray-Scott (GS) model, which was introduced for continuously stirred systems in [10]. It is given by setting $u_{b}=1, g(u)=A u, p=r=2$, and $f=-u$ in (1.1), where $A>0$ is a bifurcation parameter.

We will also consider the sub-diffusive counterpart of (1.1), formulated as

$$
\partial_{t}^{\gamma} v=\varepsilon^{2 \gamma} v_{x x}-v+g(u) v^{p}, \quad \tau \partial_{t}^{\gamma} u=u_{x x}+\left(u_{b}-u\right)+\varepsilon^{-\gamma} f(u) v^{r},
$$

where the anomaly exponent $\gamma$ is on the range $0<\gamma<1$. The regular diffusion RD system (1.1) is obtained by setting $\gamma=1$. In (1.2), the sub-diffusive operator as applied to a function $h(t)$ is defined by

$$
\frac{d^{\gamma}}{d t^{\gamma}} h(t) \equiv-\frac{1}{\Gamma(-\gamma)} \int_{0}^{t} \frac{h(t)-h(t-\zeta)}{\zeta^{\gamma+1}} d \zeta, \quad 0<\gamma<1
$$

where $\Gamma(z)$ is the Gamma function. Sub-diffusion has been observed in nature and in particular in biological systems, where diffusion is often hindered due to crowding effects of the medium. For a survey of anomalous diffusion and fractional calculus see [18], [27], and [28].

There have been many studies over the past decade of the stability and dynamics of spike-type patterns in the semi-strong spike interaction limit in a one-dimensional domain for some specific RD systems of the type (1.1) with regular diffusion including, the Gierer-Meinhardt (GM) model (cf. [39], [12], [4], [13], [38], [37], [31], [6]), the Gray-Scott (GS) model (cf. [2], [3], [20], [14], [31], [1]), the Schnakenberg model (cf. [7]), and more recently, the Brusselator model (cf. [35]), [36]) and a reaction-diffusion model of urban crime (cf. [15]). However, much less is known about how these results for specific systems extend to more general classes of RD systems. There have been only a few studies characterizing the slow dynamics and stability of pulses for classes of RD systems (cf. [5], [29], [41], [25])), a few of which are discussed below.

In the presence of sub-diffusion, there are only a few studies of the stability and dynamics of localized spike-type solutions. For the sub-diffusive GM model where $g(u)=u^{-q}, f(u)=u^{-s}$, and $u_{b}=0$ in (1.2), the slow dynamics and stability of multi-spike solutions on a finite domain was analyzed in [24]. In [25], the stability of a one-spike solution to the more general sub-diffusive problem (1.2) but with $p=2 r-3$ and $r>1$ was analyzed. For the related problem of super-diffusion, the stability and dynamics of an interface with a piecewise linear kinetics was considered in [21], and the stability and dynamics of a spike for the super-diffusive GM problem was studied in [26]. In contrast, for the simpler situation where the RD system with diffusion anomaly admits a spatially homogeneous equilibrium state in a domain with periodic boundary conditions, the stability of such states can be analyzed using a more conventional approach based on dispersion relations as derived by Fourier transform techniques (cf. [11], [30], [9], [23], [22], [34]).

In our study of (1.1) and (1.2), we will restrict our focus to the simplest scenario of interacting spikes on the infinite line. Specifically, we shall consider symmetric two-spike quasi-equilibrium patterns, corresponding to a pattern of two spikes of a common amplitude. By using the method of matched 
asymptotic expansions in the limit $\varepsilon_{0} \rightarrow 0$, we readily show that the problem of constructing these quasi-equilibrium patterns is reduced to solving for the roots of certain nonlinear algebraic equations. In addition, for both (1.1) and (1.2), we derive a finite dimensional dynamical system characterizing the slow evolution of the centers of the spikes, and we determine conditions on the nonlinearities $f$ and $g$ for which spike-interactions are either attractive or repulsive. However, the primary emphasis in our analysis is to characterize theoretically the linear stability properties of the symmetric two-spike quasi-equilibrium pattern with respect to fast $\mathcal{O}(1)$ time-scale instabilities. Our analysis shows that there are two distinct types of instabilities: competition instabilities leading to a symmetry breaking bifurcation of the twospike quasi-equilibrium pattern, and oscillatory temporal instabilities of the spike amplitudes resulting from a Hopf bifurcation. In the absence of such instabilities, the two-spike quasi-equilibrium pattern for the regular diffusion case (1.1) exhibits slow time-dependent spike dynamics, whereby the location of the spikes drift with an asymptotically small speed of order $\mathcal{O}\left(\varepsilon_{0}^{2}\right)$. For the sub-diffusive problem (1.2), the speed of spike motion is found to be asymptotically slower.

Our study of the dynamics and stability of two-spike patterns for (1.1) is most closely related to the analysis of [5] and [25]. In [5], techniques of geometric singular perturbation theory were used to analyze the existence and slow dynamics of two-spike patterns for a class of RD system closely related to (1.1). However, in [5], no analytical study of the stability properties of these two-spike quasi-equilibria to $\mathcal{O}(1)$ time-scale instabilities was undertaken. In [25], the existence of a one-spike equilibrium solution on the infinite line was analyzed for the class of RD system (1.1) and its fractional diffusive counterpart (1.2), and rigorous stability results of this solution were obtained for the exponent relation $p=2 r-3$ with $r>1$.

The main technical challenge for the study of the stability of symmetric two-spike quasi-equilibrium patterns with regular diffusion in the semi-strong interaction limit is that one must rigorously analyze the discrete spectrum of the following class of nonlocal eigenvalue problems (NLEP) for $\Phi(y)$ on the infinite line $-\infty<y<\infty$ :

$$
L_{0} \Phi-\mathcal{C}_{ \pm}(\lambda) a(w) \int_{-\infty}^{\infty} b(w) \Phi d y=\lambda \Phi, \quad-\infty<y<\infty ; \quad \Phi \rightarrow 0 \quad \text { as } \quad|y| \rightarrow \infty .
$$

Here $w(y)$ is the homoclinic of $w^{\prime \prime}-w+Q(w)=0$ for certain $Q(w)$ with $Q(0)=Q^{\prime}(0)=0, L_{0} \Phi=\Phi^{\prime \prime}-\Phi+$ $Q^{\prime}(w) \Phi$ is the linearized operator around $w, b(w)$ and $a(w)$ are nonlinear functions with $a(0)=b(0)=0$, and $\mathcal{C}_{ \pm}(\lambda)$ are two different transcendental functions of $\lambda$, one for each of the two signs. The two different choices of sign in (1.4) correspond to either a synchronous or an asynchronous instability of the spike amplitudes. This latter instability is also referred to as a competition instability (cf. [31], [38]). Since the NLEP (1.4) is non-self-adjoint and non-local, it is a difficult problem to find sufficient conditions to guarantee that all discrete eigenvalues of $(1.4)$ satisfy $\operatorname{Re}(\lambda)<0$. For simple power nonlinearities where $Q(w)=w^{p}$ with $p \geq 2, a(w)=w^{m}$ with $m>0$, and $b(w)=w^{r}$ with $r>0$, there are some rigorous results for the spectrum of (1.4) for some range of the exponents $p, m$, and $r$ (see the survey [40]). However, the theory is intricate and still incomplete.

As shown for the one-spike problem in [25], the spectrum of the associated NLEP can be determined explicitly for the sub-range of exponents $p=2 r-3$ and $r>1$ in the nonlinearity of (1.1). This was not observed in previous stability analyses (cf. [39], [4], [38], [12], [37]). In this paper we extend this recent result for the one-spike problem to the case of a symmetric two-spike quasi-equilibrium solution on the infinite line. For the sub-range $p=2 r-3$ and $r>1$ of exponents, we will derive and then analyze explicit transcendental equations for the discrete eigenvalues $\lambda$ of the associated NLEP corresponding to both the competition and synchronous instability modes. In this way, we will obtain an explicit stability theory for symmetric two-spike quasi-equilibrium solutions of (1.1). Similar stability results are obtained for the sub-diffusive problem (1.2).

The outline of this paper is as follows. In $\S 2$ a symmetric two-spike quasi-equilibrium solution for (1.1) is constructed, and in $\S 2.1$ the bifurcation point for the birth of asymmetric two-spike quasi-equilibria is calculated. In $\S 3$ a differential algebraic (DAE) system for the slow dynamics of spikes is derived for the 
regular diffusion model (1.1) and for its sub-diffusive counterpart (1.2). In $\S 4$ an NLEP characterizing the stability on an $\mathcal{O}(1)$ time-scale of this solution is derived. For $p=2 r-3$ and $r>1$, it is shown in $\S 4.1$ that the discrete spectrum of this NLEP can be obtained in terms of the roots of certain simple transcendental equations. From a study of these transcendental equations in $\S 4.2$, rigorous results for the stability and instability of symmetric two-spike quasi-equilibria for (1.1) are obtained. In $\S 4.3$, some remarks are made about the stability properties of the corresponding sub-diffusive RD system (1.2). Three explicit examples, each with qualitatively different phenomena, are given in $\S 5$ to illustrate the theory. Finally, we conclude with a brief discussion in $\S 6$.

\section{Two-Spike Quasi-Equilibrium Solutions on the Infinite Line}

We first consider the existence of two-spike quasi-equilibria to (1.1) on the infinite line. For simplicity, we consider a symmetric configuration for which the locations of the two spikes, with a common spike amplitude, are at $x=x_{0}>0$ and at $x=-x_{0}$. By symmetry, we need only consider the half-range $0 \leq x<\infty$ and impose the symmetry boundary conditions $u_{x}=v_{x}=0$ on $x=0$ in (1.1). More generally, in $\S 2.1$ below we determine the bifurcation point at which asymmetric two-spike quasi-equilibria bifurcate off the symmetric two-spike quasi-equilibrium solution branch.

We now use the method of matched asymptotic expansions to construct a symmetric two-spike quasiequilibrium solution to (1.1) in the limit $\varepsilon_{0} \ll 1$. In the inner region near $x=x_{0}>0$, we introduce the new variables $y, V$, and $U$, by

$$
y=\varepsilon_{0}^{-1}\left(x-x_{0}\right), \quad V(y)=v\left(x_{0}+\varepsilon_{0} y\right), \quad U(y)=u\left(x_{0}+\varepsilon_{0} y\right) .
$$

Upon expanding $U=U_{0}+\varepsilon_{0} U_{1}+\cdots$ and $V=V_{0}+\varepsilon_{0} V_{1}+\cdots$, and substituting into (1.1), we obtain that $U_{0}$ must be a constant and that $V_{0}$ satisfies

$$
V_{0}^{\prime \prime}-V_{0}+g_{0} V_{0}^{p}=0, \quad-\infty<y<\infty
$$

where $g_{0} \equiv g\left(U_{0}\right)$. When $g_{0}>0$, there is a unique positive homoclinic solution for $V_{0}$ given explicitly by

$$
V_{0}(y)=\frac{w(y)}{g_{0}^{1 /(p-1)}},
$$

where $w(y)$ is the unique homoclinic satisfying

$$
w^{\prime \prime}-w+w^{p}=0, \quad-\infty<y<\infty ; \quad w \rightarrow 0 \quad \text { as } \quad|y| \rightarrow \infty ; \quad w^{\prime}(0)=0, \quad w(0)>0 .
$$

The solution to (2.3a) is given explicitly by (cf. [4])

$$
w(y)=\left\{\left(\frac{p+1}{2}\right) \operatorname{sech}^{2}\left(\frac{(p-1)}{2} y\right)\right\}^{1 /(p-1)} .
$$

In the outer region, defined for $\left|x-x_{0}\right| \gg \mathcal{O}\left(\varepsilon_{0}\right)$, we obtain to all orders in $\varepsilon_{0}$ that $v=0$, and that the nonlinear term in the $u$-equation of (1.1) can be represented in terms of a Dirac mass as

$$
\varepsilon_{0}^{-1} f(u) v^{r} \rightarrow f_{0}\left(\int_{-\infty}^{\infty}\left[V_{0}(y)\right]^{r} d y\right) \delta\left(x-x_{0}\right)=\frac{f_{0} b_{r}}{g_{0}^{r /(p-1)}} \delta\left(x-x_{0}\right), \quad b_{r} \equiv \int_{-\infty}^{\infty} w^{r} d y,
$$

where we have defined $f_{0} \equiv f\left(U_{0}\right)$. In this way, we obtain that the leading-order outer solution for $u$ satisfies

$$
u_{x x}-\left(u-u_{b}\right)=-\frac{f_{0} b_{r}}{g_{0}^{r /(p-1)}} \delta\left(x-x_{0}\right), \quad 0 \leq x<\infty ; \quad u_{x}(0)=0 ; \quad u \rightarrow u_{b} \quad \text { as } \quad|x| \rightarrow \infty .
$$


The solution for $u$ can be written as

$$
u=u_{b}+\frac{f_{0} b_{r}}{g_{0}^{r /(p-1)}} G\left(x ; x_{0}\right),
$$

where $G\left(x ; x_{0}\right)$ is the Green's function satisfying

$$
G_{x x}-G=-\delta\left(x-x_{0}\right), \quad 0<x<\infty ; \quad G_{x}=0 \quad \text { on } \quad x=0 ; \quad G \rightarrow 0 \quad \text { as } \quad x \rightarrow \infty .
$$

The explicit solution for $G\left(x ; x_{0}\right)$ is

$$
G\left(x ; x_{0}\right)= \begin{cases}G\left(x_{0} ; x_{0}\right)\left(\cosh x / \cosh x_{0}\right) & 0 \leq x \leq x_{0}, \quad G\left(x_{0} ; x_{0}\right)=\frac{1}{2}\left(1+e^{-2 x_{0}}\right) . \\ G\left(x_{0} ; x_{0}\right) e^{-\left(x-x_{0}\right)} & x \geq x_{0},\end{cases}
$$

Then, to match inner and outer solutions for $u$ we must set $U_{0}=u\left(x_{0}\right)$, which yields a nonlinear algebraic equation for $U_{0}$. We summarize our asymptotic result for the symmetric two-spike quasi-equilibrium solution for (1.1) in the formal proposition as follows:

Proposition 2.1. Consider a symmetric two-spike quasi-equilibrium solution to (1.1), with spikes of a common amplitude, and centered at $x=x_{0}>0$ and $x=-x_{0}$. Then, for $\varepsilon_{0} \rightarrow 0$ this solution $v_{e}(x)$ and $u_{e}(x)$ is given asymptotically on the range $x \geq 0$ by

$$
v_{e}(x) \sim \frac{1}{g_{0}^{1 /(p-1)}} w\left(\varepsilon_{0}^{-1}\left(x-x_{0}\right)\right), \quad u_{e}(x) \sim u_{b}+\left(U_{0}-u_{b}\right) \frac{G\left(x ; x_{0}\right)}{G\left(x_{0} ; x_{0}\right)},
$$

where $w(y)$ is the homoclinic of (2.3) and $U_{0}=U_{0}\left(x_{0}\right)$ is a solution to the nonlinear algebraic equation

$$
U_{0}-u_{b}=\frac{f_{0} b_{r}}{g_{0}^{r /(p-1)}} G\left(x_{0} ; x_{0}\right), \quad f_{0} \equiv f\left(U_{0}\right), \quad g_{0} \equiv g\left(U_{0}\right), \quad b_{r} \equiv \int_{-\infty}^{\infty} w^{r} d y .
$$

Here $G$ is the Green's function satisfying (2.4). This construction is valid provided that $g_{0}=g\left(U_{0}\right)>0$ and $x_{0} \gg \mathcal{O}\left(\varepsilon_{0}\right)$. The solution is extended to $x<0$ by an even reflection about $x=0$.

The problem of characterizing the existence of a symmetric two-spike quasi-equilibrium solution to (1.1) is thereby reduced to the problem of determining the solution structure to the nonlinear algebraic problem (2.6) for different functions $f(u)$ and $g(u)$. This problem may have multiple solutions, a unique solution, or no solution, depending on the range of parameters and the choices of the kinetics $f(u)$ and $g(u)$. Explicit examples of the theory are given below in $\S 5$.

\subsection{Bifurcation to Asymmetric Two-Spike Quasi-Equilibria}

We remark that (1.1) also has asymmetric two-spike quasi-equilibrium solutions that bifurcate off the symmetric two-spike quasi-equilibrium solution branch at certain critical values of the parameters. As shown below in $\S 4.2$, these bifurcation points can also be found by linearizing (1.1) about the symmetric two-spike quasi-equilibrium solution and seeking eigenvalues $\lambda$ that cross into the right-half plane $\operatorname{Re}(\lambda)>$ 0 through the origin.

To investigate this issue, we now briefly outline the construction of asymmetric two-spike quasiequilibrium solutions for (1.1) with spikes of different amplitude that are centered at $x=x_{0}>0$ and $x=-x_{0}<0$.

In the inner solution near $x=x_{0}$ we have to leading-order that

$$
y=\varepsilon_{0}^{-1}\left(x-x_{0}\right), \quad u \sim U_{01}, \quad v \sim \frac{w(y)}{g_{01}^{1 /(p-1)}}, \quad g_{01} \equiv g\left(U_{01}\right),
$$


with a similar analysis near $x=-x_{0}$. The outer solution for $u(x)$ now satisfies

$u_{x x}-\left(u-u_{b}\right)=-\frac{f_{01} b_{r}}{g_{01}^{r /(p-1)}} \delta\left(x-x_{0}\right)-\frac{f_{02} b_{r}}{g_{02}^{r /(p-1)}} \delta\left(x+x_{0}\right), \quad-\infty<x<\infty ; \quad u \rightarrow u_{b} \quad$ as $\quad|x| \rightarrow \infty$.

The solution for $u$ can be written as

$$
u=u_{b}+\frac{f_{01} b_{r}}{g_{01}^{r /(p-1)}} G_{\infty}\left(x ; x_{0}\right)+\frac{f_{02} b_{r}}{g_{02}^{r /(p-1)}} G_{\infty}\left(x ;-x_{0}\right),
$$

where $G_{\infty}(x ; \xi)=e^{-|x-\xi|} / 2$ is the Green's function satisfying $G_{\infty x x}-G_{\infty}=-\delta(x-\xi)$ on $-\infty<x<\infty$ with $G_{\infty} \rightarrow 0$ as $|x| \rightarrow \infty$. Then, to match inner and outer solutions for $u$ we must set $U_{01}=u\left(x_{0}\right)$ and $U_{02}=u\left(-x_{0}\right)$, which yields the two nonlinear algebraic equations for $U_{01}$ and $U_{02}$ given by

$$
\begin{aligned}
& \mathcal{M}_{1}\left(U_{01}, U_{02}\right) \equiv u_{b}+\frac{f_{01} b_{r}}{g_{01}^{r /(p-1)}} G_{\infty}\left(x_{0} ; x_{0}\right)+\frac{f_{02} b_{r}}{g_{02}^{r /(p-1)}} G_{\infty}\left(x_{0} ;-x_{0}\right)-U_{01}=0, \\
& \mathcal{M}_{2}\left(U_{01}, U_{02}\right) \equiv u_{b}+\frac{f_{01} b_{r}}{g_{01}^{r /(p-1)}} G_{\infty}\left(-x_{0} ; x_{0}\right)+\frac{f_{02} b_{r}}{g_{02}^{r /(p-1)}} G_{\infty}\left(-x_{0} ;-x_{0}\right)-U_{02}=0 .
\end{aligned}
$$

By calculating the Jacobian of the nonlinear system in (2.8), we now determine whether the symmetric two-spike equilibrium solution with $U_{0 j}=U_{0}$ for $j=1,2$, where $U_{0}$ satisfies (2.6), can undergo a bifurcation to a pattern with spikes of different height. We evaluate the Jacobian matrix for (2.8) when $U_{0}=U_{0 j}$ for $j=1,2$ to obtain

$\mathcal{J} \equiv\left(\begin{array}{cc}a & b \\ b & a\end{array}\right), \quad a \equiv \frac{\partial \mathcal{M}_{1}}{\partial U_{01}}=\frac{\partial \mathcal{M}_{2}}{\partial U_{02}}=\frac{1}{2}\left(d_{0}-\frac{d_{1}}{\beta}\right)-1, \quad b \equiv \frac{\partial \mathcal{M}_{1}}{\partial U_{02}}=\frac{\partial \mathcal{M}_{2}}{\partial U_{01}}=\frac{e^{-2 x_{0}}}{2}\left(d_{0}-\frac{d_{1}}{\beta}\right)$,

where $d_{0}, d_{1}$, and $\beta$, are defined by

$$
d_{0}=\frac{f_{0}^{\prime} b_{r}}{g_{0}^{r /(p-1)}}, \quad d_{1}=\frac{r^{2} g_{0}^{\prime}}{2 g_{0}}\left(\frac{f_{0} b_{r}}{g_{0}^{r /(p-1)}}\right), \quad \beta \equiv r^{2}-2 r .
$$

The matrix $\mathcal{J}$ is singular when $a-b=0$, with eigenvector $v_{-}=(1,-1)^{t}$, and when $a+b=0$, with eigenvector $v_{+}=(1,1)^{t}$. From $(2.9 \mathrm{a})$, this yields

$$
d_{0}-\frac{d_{1}}{\beta}=\frac{2}{1 \pm e^{-2 x_{0}}}, \quad \text { with eigenvector } \quad v_{ \pm}=(1, \pm 1)^{t} .
$$

When $p=2 r-3$ and $r>2$, it is shown below in Remark 3 of $\S 4$ that the two-spike symmetric quasiequilibrium solution loses its stability when (2.10) is satisfied. At this point an asymmetric two-spike quasi-equilibrium solution bifurcates off the symmetric solution branch.

\section{Slow Dynamics of Symmetric Two-Spike Quasi-Equilibria}

For $\varepsilon_{0} \rightarrow 0$, we now use the method of matched asymptotic expansions to derive a differential equation for $x_{0}$ that characterizes the slow dynamics of the two-spike quasi-equilibrium solution for (1.1) constructed in Proposition 2.1.

In the inner region, we introduce the inner expansion and the slow time-scale $\sigma$ defined by

$$
v \sim V_{0}+\varepsilon_{0} V_{1}+\ldots, \quad u \sim U_{0}+\varepsilon_{0} U_{1}+\ldots, \quad y=\varepsilon_{0}^{-1}\left[x-x_{0}(\sigma)\right], \quad \sigma=\varepsilon_{0}^{2} t .
$$


Upon substituting this expansion into (1.1), we obtain that $U_{0}$ is independent of $y$ and that $V_{0}$ satisfies (2.1). For $\tau=\mathcal{O}(1)$, we obtain at next order that $U_{1}$ and $V_{1}$ satisfy

$$
L_{0} V_{1}=-\frac{g_{0}^{\prime}}{g_{0}^{p /(p-1)}} w^{p} U_{1}-\frac{1}{g_{0}^{1 /(p-1)}} w^{\prime} \dot{x}_{0}, \quad U_{1}^{\prime \prime}=-\frac{f_{0}}{g_{0}^{r /(p-1)}} w^{r},
$$

on $-\infty<y<\infty$, where $\dot{x}_{0} \equiv d x_{0} / d \sigma$. Here $L_{0}$ is the local operator defined by

$$
L_{0} \Phi \equiv \Phi^{\prime \prime}-\Phi+p w^{p-1} \Phi .
$$

Since $L_{0} w^{\prime}=0$, the solvability condition for the $V_{1}$ equation yields that

$$
\left(\int_{-\infty}^{\infty}\left(w^{\prime}\right)^{2} d y\right) \dot{x}_{0}=-\frac{g_{0}^{\prime}}{g_{0}} \int_{-\infty}^{\infty} U_{1} w^{p} w^{\prime} d y
$$

Upon integrating the right-hand side of (3.3) by parts twice, and then using the fact that $U_{1}^{\prime \prime}$ is an even function by (3.1), we obtain after a short calculation that

$$
\dot{x}_{0}=\frac{g_{0}^{\prime}}{2(p+1) g_{0}}\left(\frac{\int_{-\infty}^{\infty} w^{p+1} d y}{\int_{-\infty}^{\infty}\left(w^{\prime}\right)^{2} d y}\right)\left[U_{1}^{\prime}(+\infty)+U_{1}^{\prime}(-\infty)\right] .
$$

The ratio of the two integrals in (3.4) was evaluated in $\S 2$ of [13], with the result

$$
\frac{\int_{-\infty}^{\infty} w^{p+1} d y}{\int_{-\infty}^{\infty}\left(w^{\prime}\right)^{2} d y}=\frac{2(p+1)}{p-1}
$$

To calculate $U_{1}^{\prime}( \pm \infty)$, as needed in (3.4), we must match the inner expansion for $U$ to the outer solution for $u$. The outer solution for $u$ is quasi-steady, and hence is given asymptotically by the expression for $u_{e}$ given in (2.5). Therefore, the matching condition between the inner and outer solutions for $u$ is that $U_{1}^{\prime}( \pm \infty)=u_{e x}\left(x_{0}^{ \pm}\right)$, which yields

$$
U_{1}^{\prime}( \pm \infty)=\left(U_{0}-u_{b}\right) \frac{G_{x}\left(x_{0}^{ \pm} ; x_{0}\right)}{G\left(x_{0} ; x_{0}\right)}
$$

Upon substituting (3.6) and (3.5) into (3.4), we obtain that

$$
\dot{x}_{0}=\frac{g_{0}^{\prime}}{(p-1) g_{0}}\left(U_{0}-u_{b}\right) \frac{\left[G_{x}\left(x_{0}^{+} ; x_{0}\right)+G_{x}\left(x_{0}^{-} ; x_{0}\right)\right]}{G\left(x_{0} ; x_{0}\right)} .
$$

Finally, we use (2.4) to calculate the required Green's function ratio in this expression. In this way, we obtain the following formal result:

Proposition 3.1. For $\varepsilon_{0} \rightarrow 0$ and $\tau=\mathcal{O}(1)$, consider a symmetric two-spike quasi-equilibrium solution to the regular diffusion $R D$ system (1.1) with spikes at $x=x_{0}>0$ and $x=-x_{0}$, and having a common spike amplitude. Then, provided that there are no fast $\mathcal{O}(1)$ instabilities of the quasi-equilibrium profile, the slow dynamics of the spike location $x_{0}(\sigma)$ with $\sigma=\varepsilon_{0}^{2} t$ satisfies the ODE

$$
\frac{d x_{0}}{d \sigma} \sim-\frac{2}{p-1}\left(\frac{g_{0}^{\prime}}{g_{0}}\right)\left(U_{0}-u_{b}\right) \frac{e^{-2 x_{0}}}{1+e^{-2 x_{0}}}=-\frac{g_{0}^{\prime}}{(p-1) g_{0}} \frac{f_{0} b_{r}}{g_{0}^{r /(p-1)}} e^{-2 x_{0}} .
$$

Here $g_{0}=g\left(U_{0}\right), g_{0}^{\prime}=g^{\prime}\left(U_{0}\right), f_{0}=f\left(U_{0}\right), b_{r} \equiv \int_{-\infty}^{\infty} w^{r} d y$, and $U_{0}=U_{0}\left(x_{0}\right)$ satisfies the nonlinear algebraic equation in (2.6). 


\subsection{Slow Dynamics of Symmetric Two-Spike Quasi-Equilibria with Sub-Diffusion}

In this subsection we derive an ODE system for the dynamics of a two-spike quasi-equilibrium solution for the sub-diffusive system (1.2). This analysis is an extension of that in [24], where an ODE system for the sub-diffusive Gierer-Meinhardt (GM) model was derived.

We consider a symmetric two-spike quasi-equilibrium solution with spikes centered at $x=x_{0}(\sigma)>0$ and $x=-x_{0}(\sigma)<0$, where $\sigma=\varepsilon^{\alpha} t$ and $\alpha>0$ is the slow time-scale to be found. In the inner region near $x_{0}$, we introduce the inner coordinate $y \equiv \varepsilon^{-\gamma}\left[x-x_{0}(\sigma)\right]$, and the inner expansion

$V(y, \sigma)=v\left(x_{0}+\varepsilon^{\gamma} y, \varepsilon^{-\alpha} \sigma\right)=V_{0}+\varepsilon^{\gamma} V_{1}+\ldots, \quad U(y, \sigma)=u\left(x_{0}+\varepsilon^{\gamma} y, \varepsilon^{-\alpha} \sigma\right)=U_{0}(\sigma)+\varepsilon^{\gamma} U_{1}+\ldots$.

From the definition (1.3) of the fractional derivative, it follows that if we change variables as $f(t) \equiv$ $f\left(\varepsilon^{-\alpha} \sigma\right) \equiv F(\sigma)$, the fractional derivative satisfies $\partial_{t}^{\gamma} f(t)=\varepsilon^{\gamma \alpha} \partial_{\sigma}^{\gamma} F(\sigma)$. Upon using this fact, and substituting (3.8) into (1.2), we obtain that

$$
\begin{aligned}
\varepsilon^{\gamma \alpha} \partial_{\sigma}^{\gamma}\left(V_{0}+\cdots\right) & =V_{0 y y}-V_{0}+g_{0} V_{0}^{p}+\varepsilon^{\gamma}\left(V_{1 y y}-V_{1}+p g_{0} V_{0}^{p-1} V_{1}+g_{0}^{\prime} V_{0}^{p} U_{1}\right)+\mathcal{O}\left(\varepsilon^{2 \gamma}\right), \\
\varepsilon^{\gamma \alpha} \tau \partial_{\sigma}^{\gamma}\left(U_{0}+\cdots\right) & =\varepsilon^{-2 \gamma} U_{0 y y}+\varepsilon^{-\gamma}\left(U_{1 y y}+f_{0} V_{0}^{r}\right)+\mathcal{O}(1) .
\end{aligned}
$$

Here we have defined $g_{0}=g\left(U_{0}\right), g_{0}^{\prime}=g^{\prime}\left(U_{0}\right)$, and $f_{0}=f\left(U_{0}\right)$.

Assuming that $\tau=\mathcal{O}(1)$, and recalling that $0<\gamma \leq 1$ and $\alpha>0$, we obtain to leading order from (3.9) that $U_{0}=U_{0}(\sigma)$ is independent of $y$ and that $V_{0}$ is the homoclinic of (2.1) given by

$$
V_{0}(y, \sigma)=\frac{w(y)}{g_{0}^{1 /(p-1)}}
$$

where $g_{0}$ depends on $\sigma$ through $U_{0}$. With $y=\varepsilon^{-\gamma}\left[x-x_{0}(\sigma)\right]$ we then must calculate the term $\partial_{\sigma}^{\gamma} V_{0}$ on the left-hand side of (3.9a). Normally this is done by differentiating $V_{0}(y(\sigma), \sigma)$ with respect to the first argument together with an application of the chain rule involving $\frac{d x_{0}}{d \sigma}$. The derivative with respect to the second argument is of order of magnitude smaller than the first as $\varepsilon \rightarrow 0$. This procedure requires care in the case of fractional derivatives and is done with the following Lemma of [24]:

Lemma 3.2. (From [24]): Let $A(y(\sigma)) \in C^{\infty}$ with $y \equiv \varepsilon^{-\gamma}\left[x-x_{0}(\sigma)\right], x_{0} \in C^{\infty}$ and $0<\gamma<1$. Then, in the limit $\varepsilon \rightarrow 0$, the chain rule of differentiation is given by

$$
\partial_{\sigma}^{\gamma} A(y(\sigma)) \sim-\varepsilon^{-\gamma^{2}} \operatorname{sgn}\left(\frac{d x_{0}}{d \sigma}\right)\left|\frac{d x_{0}}{d \sigma}\right|^{\gamma} \mathfrak{D}_{y}^{\gamma} A(y),
$$

where $\mathfrak{D}_{y}^{\gamma} A(y)$ is defined by

$$
\mathfrak{D}_{y}^{\gamma} A(y) \equiv \operatorname{sgn}\left(\frac{d x_{0}}{d \sigma}\right) \frac{1}{\Gamma(-\gamma)} \int_{0}^{\infty}\left\{A(y)-A\left(y+\operatorname{sgn}\left(\frac{d x_{0}}{d \sigma}\right) \xi\right)\right\} \frac{d \xi}{\xi^{\gamma+1}},
$$

and $\Gamma(z)$ is the Gamma function.

Proof: : This is Lemma 2.1 of [24]. We repeat the proof of this result here for the convenience of the reader. From (1.3) we get

$$
\partial_{\sigma}^{\gamma} A(y(\sigma))=-\frac{1}{\Gamma(-\gamma)} \int_{0}^{\sigma}\left\{A\left(\frac{x-x_{0}(\sigma)}{\varepsilon^{\gamma}}\right)-A\left(\frac{x-x_{0}(\sigma-\zeta)}{\varepsilon^{\gamma}}\right)\right\} \frac{d \zeta}{\zeta^{\gamma+1}} .
$$

We then define a new variable $\xi$ in terms of $\zeta$ by

$$
\xi \equiv \varepsilon^{-\gamma}\left[x_{0}(\sigma-\zeta)-x_{0}(\sigma)\right] .
$$


Since $x_{0} \in C^{\infty}, x_{0}$ can be Taylor-expanded in order to solve for $\zeta$ in terms of $\xi$ when $\varepsilon \ll 1$. We calculate

so that (3.13) becomes

$$
x_{0}(\sigma-\zeta)=x_{0}(\sigma)-\frac{d x_{0}}{d \sigma} \zeta+\frac{1}{2} \frac{d^{2} x_{0}}{d \sigma^{2}} \zeta^{2}-\ldots,
$$

$$
\xi=\varepsilon^{-\gamma}\left(-\frac{d x_{0}}{d \sigma} \zeta+\frac{1}{2} \frac{d^{2} x_{0}}{d \sigma^{2}} \zeta^{2}-+\ldots\right) .
$$

Away from the fixed points, the series is reverted to leading order to get

$$
\zeta=-\left(\frac{d x_{0}}{d \sigma}\right)^{-1}\left(\varepsilon^{\gamma} \xi-\frac{1}{2} \frac{d^{2} x_{0}}{d \sigma^{2}} \zeta^{2}+\ldots\right), \quad \frac{d x_{0}}{d \sigma} \neq 0,
$$

and, thus, by a recursive substitution into higher powers of $\zeta$ we derive that

$$
\zeta \sim \varepsilon^{\gamma}\left(-\frac{d x_{0}}{d \sigma}\right)^{-1} \xi+\mathcal{O}\left(\varepsilon^{2 \gamma}\right)
$$

Therefore, for $\varepsilon \ll 1$, (3.12) becomes

$$
\partial_{\sigma}^{\gamma} A\left(y(\sigma) \sim-\frac{\varepsilon^{-\gamma^{2}}}{\Gamma(-\gamma)}\left(-\frac{d x_{0}}{d \sigma}\right)^{-1} \int_{0}^{-\infty \cdot \operatorname{sgn}\left(\frac{d x_{0}}{d \sigma}\right)}(A(y)-A(y-\xi))\left(-\frac{d x_{0}}{d \sigma} \frac{1}{\xi}\right)^{\gamma+1} d \xi\right.
$$

Finally, upon splitting this result into the two cases $\frac{d x_{0}}{d \sigma} \lessgtr 0$, and after changing variables to have the upper integration bound positive, the desired result is obtained.

In this way, we obtain for $\varepsilon \rightarrow 0$ that

$$
\partial_{\sigma}^{\gamma} V_{0} \sim-g_{0}^{-1 /(p-1)} \varepsilon^{-\gamma^{2}} \operatorname{sgn}\left(\frac{d x_{0}}{d \sigma}\right)\left|\frac{d x_{0}}{d \sigma}\right|^{\gamma} \mathfrak{D}_{y}^{\gamma} w(y)+\mathcal{O}(1),
$$

where the negligible $\mathcal{O}(1)$ term involves fractional $\sigma$ derivatives of $g_{0}$. Upon substituting (3.14) into (3.9a), we observe that we must choose the slow time-scale as $\alpha=\gamma+1$ in order to balance terms for $V_{1}$.

With this choice for the slow time-scale $\alpha$, we obtain that

$$
\begin{aligned}
L_{0} V_{1} & =-\frac{g_{0}^{\prime}}{g_{0}^{p /(p-1)}} w^{p} U_{1}-\frac{1}{g_{0}^{1 /(p-1)}} \operatorname{sgn}\left(\frac{d x_{0}}{d \sigma}\right)\left|\frac{d x_{0}}{d \sigma}\right|^{\gamma} \mathfrak{D}_{y}^{\gamma} w, \\
U_{1}^{\prime \prime} & =-\frac{f_{0} w^{r}}{g_{0}^{r /(p-1)}},
\end{aligned}
$$

where $L_{0}$ is the local operator defined in (3.2). In obtaining (3.15b) from (3.9b) we neglected the fractional time derivatives on the left-hand side of $(3.9 \mathrm{~b})$, which is valid when $\tau=o\left(\varepsilon^{-\gamma(2+\gamma)}\right)$. Since $0<\gamma<1$, this condition on $\tau$ holds for our assumed range $\tau=\mathcal{O}(1)$.

Since $L_{0} w^{\prime}=0$, the solvability condition for the $V_{1}$ equation of (3.15a) yields that

$$
\operatorname{sgn}\left(\frac{d x_{0}}{d \sigma}\right)\left|\frac{d x_{0}}{d \sigma}\right|^{\gamma} \int_{-\infty}^{\infty} w^{\prime} \mathfrak{D}_{y}^{\gamma} w d y=-\frac{g_{0}^{\prime}}{g_{0}} \int_{-\infty}^{\infty} U_{1} w^{p} w^{\prime} d y .
$$

Upon integrating by parts twice on the right-hand side of (3.16), and using the fact from (3.15b) that $U_{1}^{\prime \prime}$ is even, we obtain that

$$
\operatorname{sgn}\left(\frac{d x_{0}}{d \sigma}\right)\left|\frac{d x_{0}}{d \sigma}\right|^{\gamma} \int_{-\infty}^{\infty} w^{\prime} \mathfrak{D}_{y}^{\gamma} w d y=\frac{g_{0}^{\prime}}{2(p+1) g_{0}}\left(\int_{-\infty}^{\infty} w^{p+1} d y\right)\left[U_{1}^{\prime}(\infty)+U_{1}^{\prime}(-\infty)\right] .
$$

Finally, we use (3.6) to calculate $U_{1}^{\prime}( \pm \infty)$ in terms of the Green's function of (2.4). We summarize our result in the following formal proposition as follows: 
Proposition 3.3. For $\varepsilon \rightarrow 0$ and $\tau=\mathcal{O}(1)$, consider a symmetric two-spike quasi-equilibrium solution to the sub-diffusive $R D$ system (1.2) with spikes at $x=x_{0}>0$ and $x=-x_{0}$, and having a common spike amplitude. Then, provided that there are no fast $\mathcal{O}(1)$ instabilities of the quasi-equilibrium profile, the slow dynamics of the spike location $x_{0}(\sigma)$ with slow time-scale $\sigma=\varepsilon^{\gamma+1} t$ satisfies the ODE

$$
\operatorname{sgn}\left(\frac{d x_{0}}{d \sigma}\right)\left|\frac{d x_{0}}{d \sigma}\right|^{\gamma}=-\frac{g_{0}^{\prime}}{(p+1) g_{0}} h(p ; \gamma)\left(U_{0}-u_{b}\right) \frac{e^{-2 x_{0}}}{1+e^{-2 x_{0}}}=-\frac{g_{0}^{\prime}}{2(p+1) g_{0}} h(p ; \gamma) \frac{f_{0} b_{r}}{g_{0}^{r /(p-1)}} e^{-2 x_{0}},
$$

where $U_{0}=u_{0}\left(x_{0}\right)$ satisfies the nonlinear algebraic equation (2.6). In (3.18), the anomaly-dependent factor $h(p ; \gamma)$ is defined by

$$
h(p ; \gamma) \equiv\left(\int_{-\infty}^{\infty} w^{p+1} d y\right) /\left(\int_{-\infty}^{\infty} w^{\prime}(y) \mathfrak{D}_{y}^{\gamma} w d y\right)
$$

where the derivative $\mathfrak{D}_{y}^{\gamma}$ is defined in (3.11).

From (3.18), the dynamics of the spikes depends on the anomaly dependent factor $h(p ; \gamma)$. Some key properties of $h(p ; \gamma)$, as derived in $\S 2.1$ of [24], are that

$$
\left.h(p ; \gamma)\right|_{x_{0}^{\prime}>0}=\left.h(p ; \gamma)\right|_{x_{0}^{\prime}<0} p>1, \quad 0<\gamma \leq 1 ; \quad h(p ; 1)=\frac{\int_{-\infty}^{\infty} w^{p+1} d y}{\int_{-\infty}^{\infty}\left(w^{\prime}\right)^{2} d y}=2\left(\frac{p+1}{p-1}\right) .
$$

Upon using the result for $h(p ; 1)$ in (3.18) it readily follows that (3.18) recovers the result (3.7) of Proposition 3.1 for slow spike dynamics with regular diffusion. We refer the reader to $\S 2.1$ of [24] for the derivation of (3.20) and for the numerical method to compute $h(p ; \gamma)$.

In Fig. 1 we plot $h(p ; \gamma)$ versus $\gamma$ for $p=3$ and for $p=5$. The reason for these special choices of $p$ is given below in $\S 4$, when we study the nonlocal eigenvalue problem associated with $\mathcal{O}(1)$ time-scale instabilities of the quasi-equilibrium solution. For $p=3$, we observe from Fig. 1 that the minimum of $h(p ; \gamma)$ is obtained when $\gamma$ is very close to $\gamma=1$. However, for $p=5$ the curve $h(p ; \gamma)$ appears to be monotone. For $\gamma=1$, for which $h(p ; 1)=2(p+1) /(p-1), h(p ; 1)$ is a monotone decreasing function of $p$ with limiting value $\lim _{p \rightarrow \infty} h(p ; 1)=2$. The numerical results in [24] support the conjecture that $h(p ; \gamma)>0$ for all $0<\gamma<1$ and $p>1$.

Remark 1: The slow time scale for the evolution of the spikes is $\sigma=\varepsilon^{\gamma+1} t$, and, consequently, the motion of the spikes is slower under the effect of sub-diffusion than with regular diffusion. To see this, define $\varepsilon_{0}=\varepsilon^{\gamma}$ so that the diffusion coefficient of $v$ is $\varepsilon_{0}^{2}$, and the spikes have speed $\mathcal{O}\left(\varepsilon_{0}^{1+1 / \gamma}\right)$. Since $0<\gamma<1$ and $1+1 / \gamma>2$, this speed is slower than the speed $\mathcal{O}\left(\varepsilon_{0}^{2}\right)$ found in (3.7) of Proposition 3.1 for spike motion with regular diffusion.

Remark 2: As long as $0<\gamma<1$, the ODE in (3.18) comprises two separate equations, governing the motion of the spikes according to $\frac{d x_{0}}{d \sigma} \lessgtr 0$. For $\gamma=1$ the fractional operators in the definition of $h(p ; \gamma)$ approach $\frac{d}{d y}$ regardless of $\operatorname{sgn}\left(\frac{d x_{0}}{d \sigma}\right)$, and the fractional power on the left-hand side of (3.18) becomes unity, merging the two equations into a single equation for both the leftward and rightward motion of the spike.

\section{NLEP Stability Theory of the Quasi-Equilibrium Solutions}

Next, we analyze the linear stability of the symmetric two-spike quasi-equilibrium solution constructed in Proposition 2.1. More specifically, we will determine whether it can be unstable to any $\mathcal{O}(1)$ timescale instabilities. If such instabilities are present they occur on a much faster time-scale than the slow dynamics of the spikes. As such, in our stability analysis we will "freeze" the locations of the spikes. 


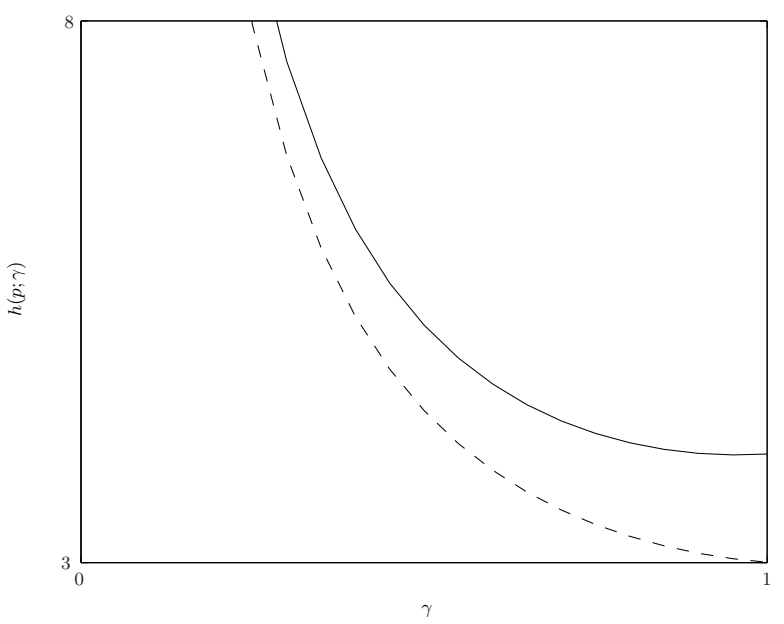

Figure 1. The function $h(p ; \gamma)$ for $p=3$ (solid curve) and for $p=5$ (dashed curve).

To analyze the linearized stability of the symmetric two-spike quasi-equilibrium solution we introduce the perturbation

$$
v=v_{e}+e^{\lambda t} \phi, \quad u=u_{e}+e^{\lambda t} \eta .
$$

Upon substituting (4.1) into (1.1) and linearizing, we obtain the eigenvalue problem

$$
\begin{gathered}
\varepsilon_{0}^{2} \phi_{x x}-\phi+p g\left(u_{e}\right) v_{e}^{p-1} \phi+g^{\prime}\left(u_{e}\right) v_{e}^{p} \eta=\lambda \phi \\
\eta_{x x}-(1+\tau \lambda) \eta=-\varepsilon_{0}^{-1}\left[f^{\prime}\left(u_{e}\right) v_{e}^{r} \eta+r f\left(u_{e}\right) v_{e}^{r-1} \phi\right] .
\end{gathered}
$$

For our symmetric two-spike case, we need only consider the half-line $0<x<\infty$, while imposing either of the two boundary conditions

$$
\phi_{x}=\eta_{x}=0 \quad \text { on } \quad x=0 \quad \text { (symmetric); } \quad \phi=\eta=0 \quad \text { on } \quad x=0 \quad \text { (asymmetric). }
$$

In particular, the imposition of $\phi_{x}=\eta_{x}=0$ on $x=0$ implies that we are seeking instabilities that are even in $x$, i.e. an in-phase or synchronous instability. In contrast, the choice $\phi=\eta=0$ on $\eta=0$ implies that we are seeking an out-of-phase or asymmetric instability. Such an instability is also called a competition instability, since the spike amplitudes are subject to a sign-fluctuating perturbation.

Since $v_{e}$ is localized near $x=x_{0}>0$, we look for a localized eigenfunction for $\phi(x)$ in the form $\phi=\Phi(y)$ where $y=\varepsilon_{0}^{-1}\left(x-x_{0}\right)$. Upon using $v_{e} \sim g_{0}^{-1 /(p-1)} w$ and $u_{e} \sim U_{0}$ for $x-x_{0}=\mathcal{O}\left(\varepsilon_{0}\right)$, we obtain from (4.2a) that $\Phi(y)$ satisfies

$$
L_{0} \Phi+\frac{g_{0}^{\prime}}{g_{0}^{p /(p-1)}} w^{p} \eta\left(x_{0}\right)=\lambda \Phi, \quad-\infty<y<\infty ; \quad \Phi \rightarrow 0 \quad \text { as } \quad|y| \rightarrow \infty,
$$

where we have labelled $g_{0}^{\prime} \equiv g^{\prime}\left(U_{0}\right)$. Here $L_{0}$ is the local operator defined in (3.2).

To derive our NLEP we must calculate $\eta\left(x_{0}\right)$ in (4.4). Since $\phi$ is localized near $x=x_{0}$, for $\varepsilon_{0} \rightarrow 0$ the right-hand side of $(4.2 \mathrm{~b})$ can be calculated in the sense of distributions by using $u_{e} \sim U_{0}$ and $v_{e} \sim g_{0}^{-1 /(p-1)} w$ as

$$
\varepsilon_{0}^{-1}\left[f^{\prime}\left(u_{e}\right) v_{e}^{r} \eta+f\left(u_{e}\right) r v_{e}^{r-1} \phi\right] \rightarrow\left[\frac{\eta\left(x_{0}\right) f_{0}^{\prime}}{g_{0}^{r /(p-1)}} \int_{-\infty}^{\infty} w^{r} d y+\frac{r f_{0}}{g_{0}^{(r-1) /(p-1)}} \int_{-\infty}^{\infty} w^{r-1} \Phi d y\right] \delta\left(x-x_{0}\right),
$$


where we have defined $f_{0}^{\prime} \equiv f^{\prime}\left(U_{0}\right)$. In this way, we obtain that the outer approximation for $\eta$ satisfies

$$
\eta_{x x}-(1+\tau \lambda) \eta=-\frac{\eta\left(x_{0}\right) f_{0}^{\prime} b_{r}}{g_{0}^{r /(p-1)}} \delta\left(x-x_{0}\right)-\frac{r f_{0}}{g_{0}^{(r-1) /(p-1)}}\left(\int_{-\infty}^{\infty} w^{r-1} \Phi d y\right) \delta\left(x-x_{0}\right), \quad-\infty<x<\infty,
$$

with $\eta \rightarrow 0$ as $x \rightarrow \infty$. At $x=0$, we impose either $\eta_{x}(0)=0$ or $\eta(0)=0$.

To represent the solution to (4.5) we introduce two new Green's functions $G_{\lambda}^{(+)}\left(x ; x_{0}\right)$ and $G_{\lambda}^{(-)}\left(x ; x_{0}\right)$, corresponding to synchronous and competition instabilities, respectively. For $x_{0}>0$, they satisfy

$$
G_{\lambda x x}^{(+)}-(1+\tau \lambda) G_{\lambda}^{(+)}=-\delta\left(x-x_{0}\right), \quad 0<x<\infty ; \quad G_{\lambda x}^{(+)}=0 \quad \text { on } \quad x=0 ; \quad G_{\lambda}^{(+)} \rightarrow 0 \quad \text { as } \quad x \rightarrow \infty,
$$

and

$$
G_{\lambda x x}^{(-)}-(1+\tau \lambda) G_{\lambda}^{(-)}=-\delta\left(x-x_{0}\right), \quad 0<x<\infty ; \quad G_{\lambda}^{(-)}=0 \quad \text { on } \quad x=0 ; \quad G_{\lambda}^{(-)} \rightarrow 0 \quad \text { as } \quad x \rightarrow \infty .
$$

A simple calculation then determines $G_{\lambda}^{( \pm)}\left(x_{0} ; x_{0}\right)$ and the ratio $G\left(x_{0} ; x_{0}\right) / G_{\lambda}^{( \pm)}\left(x_{0} ; x_{0}\right)$ as

$$
G_{\lambda}^{( \pm)}\left(x_{0} ; x_{0}\right)=\frac{1}{2 \sqrt{1+\tau \lambda}}\left(1 \pm e^{-2 x_{0} \sqrt{1+\tau \lambda}}\right), \quad \frac{G\left(x_{0} ; x_{0}\right)}{G_{\lambda}^{( \pm)}\left(x_{0} ; x_{0}\right)}=\sqrt{1+\tau \lambda}\left(\frac{1+e^{-2 x_{0}}}{1 \pm e^{-2 x_{0} \sqrt{1+\tau \lambda}}}\right)
$$

where we must take the principal value of $\sqrt{1+\tau \lambda}$. For this branch we have $\operatorname{Re}(\sqrt{1+\tau \lambda})>0$, which ensures that $G_{\lambda}^{( \pm)} \rightarrow 0$ as $x \rightarrow+\infty$. The second result in (4.6c) for the Green's function ratio is needed below for the explicit evaluation of the multiplier of the nonlocal term in the NLEP.

In terms of $G_{\lambda}^{( \pm)}$, the solution to (4.5) is

$$
\eta(x)=\frac{\eta\left(x_{0}\right) f_{0}^{\prime} b_{r}}{g_{0}^{r /(p-1)}} G_{\lambda}^{( \pm)}(x)+\frac{r f_{0}}{g_{0}^{(r-1) /(p-1)}}\left(\int_{-\infty}^{\infty} w^{r-1} \Phi d y\right) G_{\lambda}^{( \pm)}(x) .
$$

We then set $x=x_{0}$ in (4.7) and solve for $\eta\left(x_{0}\right)$. Finally, upon substituting $\eta\left(x_{0}\right)$ into (4.4) and re-writing the resulting expression by using (2.6), we obtain an NLEP for $\Phi(y)$. The result is summarized formally as follows:

Proposition 4.1. For $0<\varepsilon_{0} \ll 1$, the stability on an $\mathcal{O}(1)$ time-scale of the symmetric two-spike quasi-equilibrium solution to (1.1), as given in (2.5), is determined by the spectrum of the NLEP

$$
L_{0} \Phi-\chi w^{p}\left(\frac{\int_{-\infty}^{\infty} w^{r-1} \Phi d y}{\int_{-\infty}^{\infty} w^{r} d y}\right)=\lambda \Phi, \quad-\infty<y<\infty ; \quad \Phi \rightarrow 0 \quad \text { as } \quad|y| \rightarrow \infty .
$$

Here $L_{0}$ is the local operator, as defined in (3.2), and the multiplier $\chi=\chi(\lambda)$ of the nonlocal term has the two possible forms

$$
\chi=\chi_{ \pm} \equiv \frac{r g_{0}^{\prime}}{g_{0}}\left[\frac{f_{0}^{\prime}}{f_{0}}-\frac{1}{U_{0}-u_{b}} \frac{G\left(x_{0} ; x_{0}\right)}{G_{\lambda}^{( \pm)}\left(x_{0} ; x_{0}\right)}\right]^{-1},
$$

corresponding to either synchronous or competition instabilities of the spike amplitudes. In (4.8b), the ratio of the two Green's functions at $x=x_{0}$ is given explicitly in (4.6c). Here $U_{0}=U_{0}\left(x_{0}\right)$ is obtained from the solution to the nonlinear algebraic problem (2.6).

The continuous spectrum for (4.8a) consists of the portion $\lambda<-1$ of the negative real axis. A symmetric two-spike quasi-equilibrium solution to (1.1) is linearly stable on an $\mathcal{O}(1)$ time-scale provided that all the discrete eigenvalues of the $\operatorname{NLEP}(4.8)$ satisfy $\operatorname{Re}(\lambda) \leq 0$. A rigorous analysis of the spectrum of the NLEP in (4.8a) is very difficult owing to the fact that it is non-self-adjoint, non-local, and that $\chi$ also depends on $\lambda$.

However, as shown recently in [25], the NLEP of (4.8) is explicitly solvable for the case where $p=2 r-3$ and $r>2$. For this sub-range of exponents in the RD system (1.1), we will derive a transcendental equation for the discrete eigenvalues of (4.8a). 


\subsection{An Explicitly Solvable NLEP Problem}

We first summarize some of the known spectral properties of the local operator $L_{0}$ as obtained in [16] and [4].

Lemma 4.2. (From [4]): Let $p>1$ and suppose that $J=J(p)$ is a positive integer such that $J<$ $(p+1) /(p-1) \leq J+1$. Then, for $\phi_{l} \in \mathcal{H}^{1}(\mathbb{R})$, the local eigenvalue problem $L_{0} \phi_{l}=\nu \phi_{l}$ on $\mathbb{R}$ has $J+1$ discrete eigenvalues given by

$$
\nu_{j}=\frac{1}{4}[(p+1)-j(p-1)]^{2}-1, \quad j=0, \ldots, J .
$$

The continuous spectrum of $L_{0}$ lies in the range $-\infty<\nu<-1$.

This result is Proposition 5.6 of [4]. Notice that $\nu_{0}>0, \nu_{1}=0$, and $\nu_{j} \in(-1,0)$ for $2 \leq j \leq J$. However, $J=1$ when $p \geq 3$, so that there are no discrete eigenvalues in the interval $(-1,0)$ when $p \geq 3$. The uniqueness of a positive eigenvalue was also proved in [16].

For the special case where $p=2 r-3$ and $r>2$, it was shown recently in $[25]$ that $\phi_{l 0} \equiv w^{r-1}$ is the principal eigenfunction of $L_{0}$ associated with the unique positive eigenvalue $\nu_{0}=\beta \equiv r^{2}-2 r$. For the convenience of the reader we give the simple proof of this result.

Lemma 4.3. (From [25]): Let $p=2 r-3$ and $r>2$ so that $p>1$. For this range of $p$ and $r$, we have

$$
L_{0} w^{r-1}=\beta w^{r-1}, \quad \text { where } \quad \beta \equiv r^{2}-2 r>0 .
$$

Proof: We use $w^{\prime \prime}=w-w^{p}$ and $\left(w^{\prime}\right)^{2}=w^{2}-2 w^{p+1} /(p+1)$ to calculate

$$
\begin{aligned}
L_{0} w^{r-1} & =\left(w^{r-1}\right)^{\prime \prime}-w^{r-1}+p w^{p+r-2}, \\
& =(r-1)(r-2) w^{r-3}\left(w^{\prime}\right)^{2}+(r-1) w^{r-2} w^{\prime \prime}-w^{r-1}+p w^{p+r-2}, \\
& =(r-1)(r-2) w^{r-3}\left(w^{2}-\frac{2}{p+1} w^{p+1}\right)+(r-1) w^{r-2}\left(w-w^{p}\right)-w^{r-1}+p w^{p+r-2}, \\
& =w^{r-1}\left(r^{2}-2 r\right)+w^{r+p-2}\left(p-(r-1)-\frac{2(r-1)(r-2)}{p+1}\right) .
\end{aligned}
$$

Therefore, $L_{0} w^{r-1}=\left(r^{2}-2 r\right) w^{r-1}$ when the factor multiplying $w^{r+p-2}$ vanishes. This condition yields $p(p+1)=(r-1)[2(r-2)+(p+1)]$, which can be written as $(p-(2 r-3))(p-(1-r))=0$. Therefore, $p=2 r-3$ or $p=1-r$. Finally, we must take $p=2 r-3$ for $r>2$ in order to ensure that $p>1$.

By using the identity (4.10), we now show that the discrete spectrum of the NLEP in Proposition 4.1 can be found explicitly.

Lemma 4.4. Let $p=2 r-3$ and $r>2$, so that $p>1$, and consider the NLEP

$$
L_{0} \Phi-\chi w^{p} \frac{\int_{-\infty}^{\infty} w^{r-1} \Phi d y}{\int_{-\infty}^{\infty} w^{r} d y}=\lambda \Phi, \quad-\infty<y<\infty ; \quad \Phi \rightarrow 0, \quad|y| \rightarrow \infty
$$

with $\chi=\chi(\lambda)$. Then, for eigenfunctions $\Phi$ for which $\int_{-\infty}^{\infty} w^{r-1} \Phi d y \neq 0$, the discrete eigenvalues must satisfy the transcendental equation

$$
\lambda=\beta-\left(\frac{r}{2}\right) \chi,
$$

where $\chi=\chi(\lambda)$. The remaining eigenfunctions $\Phi$, for which $\int_{-\infty}^{\infty} w^{r-1} \Phi d y=0$, belong to the set of eigenfunctions of $L_{0}$ consisting of the zero eigenvalue and any negative real eigenvalues. 
Proof: To establish (4.12) we use Green's identity on $w^{r-1}$ and $\Phi$. Since $L_{0}$ is self-adjoint, we integrate by parts and use the decay at infinity to conclude that $\int_{-\infty}^{\infty}\left(w^{r-1} L_{0} \Phi-\Phi L_{0} w^{r-1}\right) d y=0$. From (4.10) and (4.11) with $p=2 r-3$ we then calculate that

$$
0=\int_{-\infty}^{\infty}\left(w^{r-1} L_{0} \Phi-\Phi L_{0} w^{r-1}\right) d y=\left(\chi \frac{\int_{-\infty}^{\infty} w^{3 r-4} d y}{\int_{-\infty}^{\infty} w^{r} d y}+\lambda-\beta\right) \int_{-\infty}^{\infty} w^{r-1} \Phi d y
$$

Therefore, for eigenfunctions for which $\int_{-\infty}^{\infty} w^{r-1} \Phi d y \neq 0$, we get

$$
\lambda=\beta-\chi \frac{\int_{-\infty}^{\infty} w^{3 r-4} d y}{\int_{-\infty}^{\infty} w^{r} d y}
$$

To calculate the integral ratio in (4.13), we multiply $w^{\prime \prime}-w+w^{2 r-3}=0$ by $w^{r-1}$ and $L_{0} w^{r-1}=\beta w^{r-1}$ by $w$. Upon subtracting the resulting two expressions we obtain

$$
w\left(w^{r-1}\right)^{\prime \prime}-w^{r-1} w^{\prime \prime}+(2 r-4) w^{3 r-4}=\beta w^{r} .
$$

Upon integrating this expression over $-\infty<y<\infty$ and using $w \rightarrow 0$ as $|y| \rightarrow \infty$, we obtain that $(2 r-4) \int_{-\infty}^{\infty} w^{3 r-4} d y=\beta \int_{-\infty}^{\infty} w^{r} d y$. Therefore, since $\beta=r^{2}-2 r$, the integral ratio in (4.13) is simply $\int_{-\infty}^{\infty} w^{3 r-4} d y=\left(\int_{-\infty}^{\infty} w^{r} d y\right)(r / 2)$. This yields (4.12).

Next, consider the eigenfunctions for which $\int_{-\infty}^{\infty} w^{r-1} \Phi d y=0$. From (4.11), the facts that $w^{r-1}$ is the unique and one-signed principal eigenfunction of $L_{0}$, and that any eigenfunctions of the selfadjoint operator $L_{0}$ must be orthogonal, it follows that these other eigenfunctions must belong to the set eigenfunctions of $L_{0}$ corresponding to the zero eigenvalue and any negative real eigenvalues of $L_{0}$.

This result shows that when $p=2 r-3$ and $r>2$, the zero eigenvalue and any negative real eigenvalues of $L_{0}$ remain at fixed locations in $\operatorname{Re}(\lambda) \leq 0$ independent of the multiplier $\chi$ of the nonlocal term. Therefore, to analyze whether (4.11) has any eigenvalues in $\operatorname{Re}(\lambda)>0$, we only need to analyze the roots of (4.12).

\subsection{NLEP Stability Theory with Regular Diffusion}

By combining (4.6c), (4.8b), and (4.12), we obtain explicit transcendental equations for the discrete eigenvalues of (4.8) corresponding to either synchronous or competition instabilities. The result is as follows.

Lemma 4.5. Let $p=2 r-3$ and $r>2$. Then, any discrete eigenvalue $\lambda$ of (4.8) with eigenfunction satisfying $\int_{-\infty}^{\infty} \Phi w^{r-1} d y \neq 0$, must be a root of either of the two transcendental equations

$$
\mathcal{F}_{ \pm}(\lambda)=\mathcal{G}(\lambda)
$$

where the + and - signs correspond to synchronous and competition instabilities, respectively. In (4.14a), $\mathcal{G}(\lambda)$ and $\mathcal{F}_{ \pm}(\lambda)$ are defined by

$$
\mathcal{G}(\lambda) \equiv d_{0}-\frac{d_{1}}{\beta-\lambda}, \quad \mathcal{F}_{ \pm}(\lambda) \equiv \frac{2 \sqrt{1+\tau \lambda}}{1 \pm e^{-2 x_{0} \sqrt{1+\tau \lambda}}},
$$

while the $\lambda$-independent constants $d_{0}, d_{1}$, and $\beta$, are defined by

$$
d_{0} \equiv \frac{f_{0}^{\prime} b_{r}}{g_{0}^{r /(2 r-4)}}, \quad d_{1} \equiv \frac{r^{2} g_{0}^{\prime}}{2 g_{0}}\left(\frac{f_{0} b_{r}}{g_{0}^{r /(2 r-4)}}\right), \quad \beta=r^{2}-2 r>0 .
$$

Here $f_{0} \equiv f\left(U_{0}\right), g_{0} \equiv g\left(U_{0}\right), f_{0}^{\prime} \equiv f^{\prime}\left(U_{0}\right), g_{0}^{\prime} \equiv g^{\prime}\left(U_{0}\right), b_{r} \equiv \int_{-\infty}^{\infty} w^{r} d y$, and $U_{0}=U_{0}\left(x_{0}\right)$ is the solution to the nonlinear algebraic equation (2.6). For the range of $x_{0}$ with $x_{0}>0$ for which the roots $\lambda$ to (4.14b) 
are in the stable left-half plane $\operatorname{Re}(\lambda)<0$, the slow two-spike dynamics (3.7) of Proposition 3.1 is valid. In terms of $d_{1}$, the dynamics (3.7) for $r>2$ can be written as

$$
\frac{d x_{0}}{d t}=-\frac{\varepsilon_{0}^{2} d_{1}}{r^{2}(r-2)} e^{-2 x_{0}} .
$$

If $d_{1}<0$ for any sub-range of $0<x_{0}<\infty$, the two-spike dynamics is repulsive on this sub-range. Moreover, if $d_{1}<0$ is defined for $x_{0} \rightarrow \infty$ with a finite limiting value $\lim _{x_{0} \rightarrow \infty} d_{1}=d_{1 \infty}$, then for $t \rightarrow \infty$ we obtain from (4.15) that

$$
x_{0} \sim \frac{1}{2} \log \left(\frac{2 \varepsilon_{0}^{2} t\left|d_{1 \infty}\right|}{r^{2}(r-2)}\right) .
$$

Next, we analyze the roots of (4.14a). Our first result is for the case $\tau=0$.

Proposition 4.6. Let $\tau=0$ in (4.14). For a given $x_{0}>0$, suppose that (2.6) has a solution $U_{0}\left(x_{0}\right)$. Then, (4.14a) has no roots in $\operatorname{Re}(\lambda)>0$ whenever

$$
d_{1}<0 \quad \text { and } \quad d_{0}<\mathcal{F}_{ \pm}(0)<\mathcal{G}(0)=d_{0}-\frac{d_{1}}{\beta} .
$$

As a consequence, the NLEP (4.8) has no unstable eigenvalues at $x_{0}$ when (4.17) holds. If (4.17) holds for all $x_{0}$ on some sub-range of $0<x_{0}<\infty$, there are no $\mathcal{O}(1)$ time-scale instabilities of the two-spike quasi-equilibrium solution on this sub-range. Consequently, the slow repulsive spike dynamics (4.15) is valid on the sub-range of $x_{0}>0$ for which (4.17) holds.

Proof: We first note that since $\mathcal{F}_{ \pm}(\lambda)$ depends on the product $\tau \lambda$, we have $\mathcal{F}_{ \pm}(\lambda)=\mathcal{F}_{ \pm}(0)=$ $2 /\left(1 \pm e^{-2 x_{0}}\right)$ when $\tau=0$. We first prove that (4.14a) has no real positive roots. Since $d_{1}<0$, then $\mathcal{G}(\lambda)$ in (4.14b) satisfies $\mathcal{G}^{\prime}(\lambda)>0$ for all $\lambda>0$ with $\mathcal{G}(\lambda) \rightarrow+\infty$ as $\lambda \rightarrow \beta^{-}$. In addition, $\mathcal{G}(\lambda) \rightarrow-\infty$ as $\lambda \rightarrow \beta^{+}$with $\mathcal{G}(\lambda) \rightarrow d_{0}$ as $\lambda \rightarrow \infty$. Therefore, when $d_{0}<\mathcal{F}_{ \pm}(0)<\mathcal{G}(0)$, then (4.14a) has no real positive roots.

Next, we show that there are no complex-valued roots to $(4.14 \mathrm{a})$ in $\operatorname{Re}(\lambda)>0$. This is done by using a winding number criterion to count the number $N$ of roots of $(4.14 \mathrm{a})$ in $\operatorname{Re}(\lambda)>0$. We define $\mathcal{K}_{ \pm}(\lambda)=\mathcal{F}_{ \pm}(0)-\mathcal{G}(\lambda)$, and note that $\mathcal{G}(\lambda)$ has a simple pole in $\operatorname{Re}(\lambda)>0$ at $\lambda=\beta$. We then calculate the winding number of $\mathcal{K}_{ \pm}(\lambda)$ over the counterclockwise contour consisting of the imaginary axis $-i R \leq \operatorname{Im}(\lambda) \leq i R$ and the semi-circle $\Gamma_{R}$, given by $|\lambda|=R>0$, for $-\pi / 2 \leq \arg \lambda \leq \pi / 2$. Since $\mathcal{K}_{ \pm}(\lambda) \sim \mathcal{F}_{ \pm}(0)$ as $|\lambda| \rightarrow \infty$ on $\Gamma_{R}$, it follows that there is no change in the argument of $\mathcal{K}_{ \pm}$over $\Gamma_{R}$ as $R \rightarrow \infty$. By using the argument principle, together with $\mathcal{K}_{ \pm}(\bar{\lambda})=\overline{\mathcal{K}_{ \pm}(\lambda)}$, we obtain that

$$
N=1+\frac{1}{\pi}\left[\arg \mathcal{K}_{ \pm}\right]_{\Gamma_{I}}
$$

where $\left[\arg \mathcal{K}_{ \pm}\right]_{\Gamma_{I}}$ denotes the change in the argument of $\mathcal{K}_{ \pm}$along the semi-infinite imaginary axis $\Gamma_{I}=$ $i \lambda_{I}, 0 \leq \lambda_{I}<\infty$, traversed in the downwards direction.

To calculate $\left[\arg \mathcal{K}_{ \pm}\right]_{\Gamma_{I}}$ we set $\lambda=i \lambda_{I}$ in $\mathcal{K}_{ \pm}(\lambda)=\mathcal{F}_{ \pm}(0)-\mathcal{G}(\lambda)$ and separate real and imaginary parts to get

$$
\mathcal{K}_{ \pm}\left(i \lambda_{I}\right)=\mathcal{K}_{R \pm}\left(\lambda_{I}\right)+i \mathcal{K}_{I \pm}\left(\lambda_{I}\right) ; \quad \mathcal{K}_{R \pm}(\lambda) \equiv \mathcal{F}_{ \pm}(0)-d_{0}+\frac{d_{1} \beta}{\beta^{2}+\lambda_{I}^{2}}, \quad \mathcal{K}_{I \pm}(\lambda) \equiv \frac{d_{1} \lambda_{I}}{\beta^{2}+\lambda_{I}^{2}}
$$

From (4.17), we calculate $\mathcal{K}_{ \pm}(0)=\mathcal{F}_{ \pm}(0)-\mathcal{G}(0)<0$ and $\mathcal{K}_{ \pm}(i \infty)=\mathcal{F}_{ \pm}(0)-d_{0}>0$. Moreover, $\mathcal{K}_{I \pm}\left(\lambda_{I}\right)<0$ for all $\lambda_{I}>0$ since $d_{1}<0$. Therefore, it follows that $\left[\arg \mathcal{K}_{ \pm}\right]_{\Gamma_{I}}=-\pi$. This gives $N=0$ from (4.18), so that the NLEP (4.8) has no unstable eigenvalues.

The second result is for the case $\tau \geq 0$ and guarantees the existence of a positive real root of (4.14a). 
Proposition 4.7. Let $\tau \geq 0$ in (4.14) and suppose $d_{1}<0$. For a given $x_{0}>0$, suppose that (2.6) has a solution $U_{0}\left(x_{0}\right)$ at which either

$$
\mathcal{F}_{+}(0)>\mathcal{G}(0)=d_{0}-\frac{d_{1}}{\beta} \quad \text { or } \quad \mathcal{F}_{-}(0)>\mathcal{G}(0)=d_{0}-\frac{d_{1}}{\beta} .
$$

Then, for any $\tau \geq 0$, the NLEP (4.8) has an unstable real positive eigenvalue at the given $x_{0}>0$.

Proof: For any $\tau \geq 0$, it is readily shown from $(4.14 \mathrm{~b})$ that $\mathcal{F}_{ \pm}(\lambda)$ is a monotone increasing function of $\lambda$ in $\lambda>0$. In addition, for $d_{1}<0$ we have $\mathcal{G}(\lambda) \rightarrow+\infty$ as $\lambda \rightarrow \beta^{-}$. Therefore, if $\mathcal{F}_{ \pm}(0)>\mathcal{G}(0),(4.14 \mathrm{a})$ must have a root in $0<\lambda<\beta$.

Remark 3: The $N L E P$ has a zero eigenvalue for all $\tau \geq 0$ when $\mathcal{F}_{ \pm}(0)=\mathcal{G}(0)$. This coincides precisely with the bifurcation point, given in (2.10) of $\S 2$, for which an asymmetric two-spike quasi-equilibrium solution bifurcates off the symmetric two-spike quasi-equilibrium solution branch.

The next result establishes sufficient conditions for the existence of a Hopf bifurcation of the two-spike quasi-equilibrium solution as $\tau$ is increased.

Proposition 4.8. For a given $x_{0}>0$, suppose that (2.6) has a solution $U_{0}\left(x_{0}\right)$ at which (4.17) holds. Then, both the synchronous and competition modes must each undergo a Hopf bifurcation at some (minimum) critical values $\tau_{H+}\left(x_{0}\right)$ and $\tau_{H-}\left(x_{0}\right)$, respectively. For $0<\tau<\tau_{H m}=\min \left(\tau_{H+}, \tau_{H-}\right)$ there are no roots of (4.14a) in $\operatorname{Re}(\lambda)>0$.

Proof: Suppose that (4.17) holds. Then, as shown in the proof of Proposition 4.6, (4.14a) has no roots in $\operatorname{Re}(\lambda)>0$ when $\tau=0$. In contrast, for $\tau>0$ sufficiently large, we now show for either the synchronous or competition mode, i.e. for either choice of sign in (4.14a), that (4.14a) has exactly two roots on the sub-range $0<\lambda<\beta$ of the positive real $\lambda$ axis. This is established by readily verifying that $\mathcal{F}_{ \pm}(\lambda)$ in (4.14b) satisfies $\mathcal{F}_{ \pm}^{\prime}(\lambda)>0$ and $\mathcal{F}_{ \pm}^{\prime \prime}(\lambda)<0$ with $\mathcal{F}_{ \pm}^{\prime}(\lambda) \rightarrow+\infty$ as $\tau \rightarrow \infty$ for any $\lambda>0$. Since $\mathcal{G}^{\prime}(\lambda)>0$ and $\mathcal{G}^{\prime \prime}(\lambda)>0$ on $0<\lambda<\beta$, it follows that when $\mathcal{F}_{ \pm}(0)<\mathcal{G}(0)$ the transcendental equation (4.14a) has exactly two roots on $0<\lambda<\beta$ for either the synchronous or competition mode. Finally, we notice that a root of (4.14a) cannot enter $\operatorname{Re}(\lambda)>0$ by crossing through the origin as $\tau$ is increased. Therefore, it follows from continuity that, for either the synchronous or competition mode, there must be critical values $\tau_{H \pm}$ of $\tau>0$ for which (4.14a) has a complex conjugate pair of pure imaginary roots, with the property that $\operatorname{Re}(\lambda)<0$ for all $\tau<\min \left(\tau_{H+}, \tau_{H-}\right)$.

Although Proposition 4.8 proves the existence of a Hopf bifurcation for the NLEP (4.8), it does not prove that $\tau_{H \pm}$ are uniquely determined. All that one can guarantee is that there must be an odd number of transversal crossings of the imaginary axis $\operatorname{Re}(\lambda)=0$ as $\tau$ ranges over $0<\tau<\infty$.

Our final result rules out the possibility of attractive two-spike dynamics that are stable to $\mathcal{O}(1)$ time-scale instabilities when $\tau>0$.

Proposition 4.9. For a given $x_{0}>0$, suppose that (2.6) has a solution $U_{0}\left(x_{0}\right)$. Then, if $d_{1}>0$ at the given $x_{0}$, the $\operatorname{NLEP}(4.8)$ has an unstable real eigenvalue for any $\tau>0$ at $x_{0}$. However, if $\tau=0, d_{1}>0$, and $\mathcal{G}(0)<\mathcal{F}_{ \pm}(0)<d_{0}$, then the $N L E P$ (4.8) has no eigenvalues in $\operatorname{Re}(\lambda)>0$.

Proof: When $d_{1}>0$, we conclude that $\mathcal{G}(\lambda)$ in $(4.14 \mathrm{~b})$ satisfies $\mathcal{G}^{\prime}(\lambda)<0$ on $0<\lambda<\beta$ with $\mathcal{G}(\lambda) \rightarrow-\infty$ as $\lambda \rightarrow \beta^{-}$. On the range $\lambda>\beta$, we have $\mathcal{G}^{\prime}(\lambda)<0$ with $\mathcal{G}(\lambda) \rightarrow+\infty$ as $\lambda \rightarrow \beta^{+}$and $\mathcal{G}(\lambda) \rightarrow d_{0}$ as $\lambda \rightarrow \infty$. Therefore, if $\tau=0$ and $\mathcal{G}(0)<\mathcal{F}_{ \pm}(0)=2 /\left(1 \pm e^{-2 x_{0}}\right)<d_{0}$, there are no roots of (4.14a) on the positive real axis. By a slight modification of the winding number proof of Proposition 4.6, it readily follows that there are no complex-valued eigenvalues in $\operatorname{Re}(\lambda)>0$ when $\tau=0$ and $\mathcal{G}(0)<\mathcal{F}_{ \pm}(0)<d_{0}$. However, since $\mathcal{F}_{ \pm}^{\prime}(\lambda)>0$ for all $\lambda>0$ and $\mathcal{F}_{ \pm}(\lambda) \rightarrow \infty$ as $\lambda \rightarrow \infty$ for any $\tau>0$, it follows that (4.14a) must have a root in $\lambda>\beta$ regardless of the initial value $\mathcal{F}_{ \pm}(0)$. Therefore, if $d_{1}>0$ at a given $x_{0}$, the $\operatorname{NLEP}$ (4.8) must have an unstable real eigenvalue for any $\tau>0$. 


\subsection{NLEP Stability Theory with Sub-Diffusion}

In this subsection we make some remarks on the stability of a two-spike quasi-equilibrium solution to the sub-diffusive RD system (1.2).

In the context of fractional differential equations, perturbations of a steady-state solution do not grow exponentially in time with a constant growth rate. Therefore, the linearized "eigenvalue problem" no longer has its classical meaning. Instead, it should be regarded as an asymptotic theory of perturbations that evolve exponentially in time to leading order as

$$
v \sim v_{e}+e^{\lambda t} \phi(x), \quad u \sim u_{e}+e^{\lambda t} \eta ; \quad \lambda(t) \sim \lambda^{(0)}+\varepsilon^{\gamma} \lambda^{(1)}(t)+\cdots .
$$

For convenience, we shall refer to $\lambda^{(0)}$ as an "eigenvalue" for the linearization of the sub-diffusive system $(1.2)$.

Upon substituting (4.20) into (1.2), and then collecting the leading order terms, we obtain the following expression involving the fractional derivative:

$$
\mathcal{S} \equiv e^{-\lambda^{(0)} t} \frac{d^{\gamma}}{d t^{\gamma}} e^{\lambda^{(0)} t}=-\frac{1}{\Gamma(-\gamma)} \int_{0}^{t} \frac{1-e^{-\lambda^{(0)} \zeta}}{\zeta^{\gamma+1}} d \zeta .
$$

In the limit $t \rightarrow \infty$ the integral converges if and only if $\operatorname{Re}\left(\lambda^{(0)}\right) \geq 0$. In the sub-diffusive case with $0<\gamma<1$ and $\lambda^{(0)}>0$ the derivative $\frac{d^{\gamma}}{d t^{\gamma}} e^{\lambda^{(0)} t}$ diverges exponentially at $t \rightarrow \infty$, but the factor $\exp \left(-\lambda^{(0)} t\right)$ makes the expression finite. However for $\lambda^{(0)}<0$ the derivative $\frac{d^{\gamma}}{d t^{\gamma}} e^{\lambda^{(0)} t}$ decays only algebraically, and with the exponential factor $\exp \left(-\lambda^{(0)} t\right)$ the expression is divergent. Therefore, since we require that $\operatorname{Re}\left(\lambda^{(0)}\right) \geq 0$, we can only study the onset of an instability, i.e. the limit $\operatorname{Re}\left(\lambda^{(0)}\right) \rightarrow 0^{+}$, but are unable to track any eigenvalues in the left half of the complex plane. This is summarized in the following lemma.

Lemma 4.10. In the limit $t=\sigma \varepsilon^{-(\gamma+1)} \gg 1$ with $\varepsilon \rightarrow 0$ and $\sigma \sim \mathcal{O}(1)$ the asymptotic relation

$$
\mathcal{S} \equiv e^{-\lambda^{(0)} t} \frac{d^{\gamma}}{d t^{\gamma}} e^{\lambda^{(0)} t} \sim \lambda^{(0)^{\gamma}}+\mathcal{O}\left(\varepsilon^{\gamma(\gamma+1)}\right)
$$

holds if and only if $\operatorname{Re}\left(\lambda^{(0)}\right) \geq 0$.

The proof of this result follows from a detailed contour integration and was given in Lemma 3.1 of [24] (and also Lemma 2.16 of [25]). From this Lemma, it follows that the spectrum of the NLEP associated with the linearization of (1.2) is determined by the roots of (4.14) where $\lambda$ is now replaced by $\lambda^{(0)^{\gamma}}$.

The results above in $\S 4.2$ for the roots of (4.14) can then be readily used to infer stability or instability for the sub-diffusive case. More specifically, if we write $\lambda=|\lambda| \exp (\imath \phi)$ with $\phi=\arg \lambda \in(-\pi, \pi]$, then the map $\lambda^{(0)^{\gamma}}=\lambda$ yields that

$$
\lambda^{(0)}=|\lambda|^{1 / \gamma} e^{\imath \phi / \gamma},
$$

where the constraint $-\pi<\phi / \gamma<\pi$ must hold in order to remain on the principal branch in the $\lambda^{(0)}$ plane. From Lemma 4.10, the derivation of the NLEP for the sub-diffusive system required that $\operatorname{Re}\left(\lambda^{(0)}\right) \geq 0$ and $\left|\lambda^{(0)}\right| \gg \mathcal{O}(\varepsilon)$. Therefore, in terms of the spectral $\lambda$ plane associated with the regular NLEP, the sub-diffusive system will be unstable if the regular NLEP (4.8) has an eigenvalue in the wedge-shaped domain of instability defined by

$$
-\frac{\pi \gamma}{2} \leq \phi \leq \frac{\pi \gamma}{2}, \quad|\lambda| \gg \mathcal{O}(\varepsilon) .
$$

This subset of the right half-plane $\operatorname{Re}(\lambda) \geq 0$ becomes narrower as $\gamma$ decreases, and concentrates on the positive real axis in the $\lambda$-plane as $\gamma \rightarrow 0^{+}$. A schematic plot of the wedge of instability is shown in Fig. 2. 


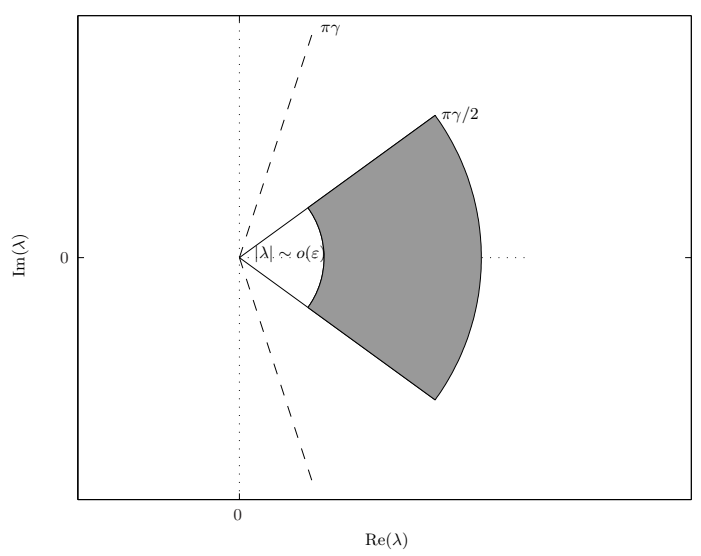

FiguRE 2. Admissibility and instability region in the $\lambda$ plane ( shaded ) corresponding to the asymptotics validity constraint $|\lambda| \sim \mathcal{O}(\varepsilon)$ or larger. The dashed lines show the region conforming to the principal branch.

In this way, we conclude that if the regular NLEP admits a positive real eigenvalue $\lambda^{*}>0$, this eigenvalue must lie in the wedge of instability for the sub-diffusive NLEP for any $\gamma$ in $0<\gamma<1$. Such an eigenvalue $\lambda^{(0)}=\left(\lambda^{*}\right)^{1 / \gamma}>0$ then yields an exponentially growing perturbation (to leading order) to the symmetric two-spike quasi-equilibrium solution of (1.2). Consequently, the instability results of Propositions 4.7 and 4.9 above, which result from a positive real eigenvalue of the regular diffusion NLEP, still hold for the sub-diffusion problem for all $\gamma$ in $0<\gamma<1$.

Next, we consider Hopf bifurcations associated with the sub-diffusive RD system (1.2) under the conditions (4.17) on $d_{0}$ and $d_{1}$. The key observation is that an unstable eigenvalue of the regular NLEP only generates an instability for the sub-diffusive NLEP when it lies within the wedge of instability (4.23). As remarked above, this wedge of instability becomes narrower as $\gamma$ decreases, and concentrates on the positive real axis in the $\lambda$-plane as $\gamma \rightarrow 0$. The anomaly-dependent Hopf bifurcation thresholds for the competition and synchronous instability modes, labelled by $\tau_{H \pm \gamma}$, are computed numerically from the implicit condition that

$$
\arg \lambda=\frac{\pi \gamma}{2}
$$

which involves the eigenvalue path $\lambda_{ \pm}=\lambda_{ \pm}(\tau)$ of the roots of (4.14) with $\operatorname{Re}(\lambda)>0$ and $\operatorname{Im}(\lambda)>0$. The eigenvalue path, for each choice of sign, crosses the border of the wedge of instability at the point where the condition (4.24) is satisfied. In this sense, we conclude that the sub-diffusive RD system (1.2) undergoes an oscillatory instability at a larger value of $\tau$ than for the case of regular diffusion.

A more explicit result for the sub-diffusive Hopf bifurcation under the condition (4.17) is difficult to obtain because of two factors: the possible non-uniqueness of values $\tau_{H \pm}$ of $\tau$ for which (4.14) has a complex conjugate pair of imaginary eigenvalues, and the possible non-monotonicity of $\operatorname{Re}\left(\lambda_{ \pm}(\tau)\right)$ and $\operatorname{Im}\left(\lambda_{ \pm}(\tau)\right)$ as $\tau$ increases. More specifically, it is theoretically possible that an eigenvalue path can first enter the wedge of instability at some critical value of $\tau$, then leave at some later value, only to re-appear within the wedge for some sufficiently large value of $\tau$. An explicit result for the sub-diffusive Hopf bifurcation is available under the assumption that, for each choice of sign, there is a unique value $\tau_{H \pm}$ of $\tau$ for which (4.14) has a complex conjugate pair of imaginary eigenvalues, and that the corresponding eigenvalue path $\lambda_{ \pm}(\tau)$ satisfies a monotonicity condition in the sense that $\operatorname{Re}\left(\lambda_{ \pm}(\tau)\right)$ is increasing, while $\operatorname{Im}\left(\lambda_{ \pm}(\tau)\right)$ is decreasing, as $\tau$ increases beyond $\tau_{H \pm}$. Under these assumptions, we can readily conclude for all $\gamma$ in $0<\gamma<1$, and for each of the two possible signs, that the Hopf bifurcation threshold $\tau_{H \gamma \pm}$ for the sub-diffusive system (1.2) must lie in the interval $\tau_{H \pm}<\tau_{H \gamma \pm}<\tau_{t \pm}$. Here $\tau_{H \pm}$ is the Hopf bifurcation threshold for the regular diffusion case, and $\tau_{t \pm}$ is the value of $\tau$ where the complex conjugate 
eigenvalue pair for the regular NLEP merges onto the positive real axis. This critical value $\tau_{t \pm}$ is the unique value of $\tau$ where the curves $\mathcal{F}_{ \pm}(\lambda)$ and $\mathcal{G}(\lambda)$ in (4.14) intersect tangentially at some point $\lambda^{*}$ on the positive real axis under the condition (4.17). In particular, $\tau_{H \gamma \pm} \longrightarrow \tau_{H \pm}$ as $\gamma \longrightarrow 1^{-}$and $\tau_{H \gamma \pm} \longrightarrow \tau_{t \pm}$ as $\gamma \longrightarrow 0^{+}$.

\section{Three Examples of the Theory}

Three explicit examples, each with qualitatively different behaviour, are now given to illustrate our main stability and instability results for RD systems of the form (1.1) with regular diffusion.

GM Model: We first consider the GM model where $g(u)=u^{-q}, f(u)=u^{-s}, u_{b}=0$, with $q>0$. Then, from the definitions in $(4.14 \mathrm{c})$, we calculate $d_{0}, d_{1}$, and $\mathcal{G}(0)$, as

$$
d_{0}=-\frac{2 s}{1+e^{-2 x_{0}}}, \quad d_{1}=-\frac{q r^{2}}{1+e^{-2 x_{0}}}, \quad \mathcal{G}(0) \equiv \frac{2}{1+e^{-2 x_{0}}}(\xi+1), \quad \xi \equiv \frac{q r}{2 r-4}-(s+1) .
$$

Since $d_{1}<0$, the two-spike dynamics is repulsive for all $x_{0}$ and the Propositions 4.6-4.8 apply. From the nonlinear algebraic equation (2.6), we determine $U_{0}$ as $U_{0}^{-\xi}=b_{r}\left(1+e^{-2 x_{0}}\right) / 2$ where $b_{r}=\int_{-\infty}^{\infty} w^{r} d y$.

For $\tau=0$, the criterion $d_{0}<\mathcal{F}_{+}(0)<\mathcal{G}(0)$ from (4.17) for stability with respect to synchronous perturbations is satisfied when $-2 s<2<2(\xi+1)$. We conclude that the synchronous mode is stable when $s>-1$ and $\xi>0$.

With regards to the competition mode when $\tau=0$, the stability criterion of (4.17) holds when both $\mathcal{F}_{-}(0)>d_{0}$ and $\mathcal{F}_{-}(0)<\mathcal{G}(0)$ are satisfied. When $s>-1$, the first inequality holds for all $x_{0}>0$, but the second inequality holds only if $\operatorname{coth}\left(x_{0}\right)<\xi+1$. This implies that for $\tau=0$, we have stability with respect to the competition mode when $x_{0}>x_{0-}$, where

$$
x_{0-} \equiv \frac{1}{2} \log \left(\frac{2+\xi}{\xi}\right), \quad \xi \equiv \frac{q r}{2 r-4}-(s+1)>0 .
$$

For $\tau=0$, we conclude that a two-spike quasi-equilibrium solution to the GM model is stable when the inter-spike separation exceeds $2 x_{0-}$, where $x_{0-}>0$ is given in (5.2).

From Proposition 4.7, a two-spike quasi-equilibrium solution is unstable for all $\tau \geq 0$ when $0<x_{0}<$ $x_{0-}$. For the range $x_{0}>x_{0-}$, Proposition 4.8 proves that both the synchronous and competition modes must undergo a Hopf bifurcation as $\tau$ is increased. At each $x_{0}$ on $x_{0}>x_{0-}$, the Hopf bifurcation threshold $\tau_{H m}\left(x_{0}\right)$ is the minimum of these two thresholds. For $r=3, q=2$, and $s=0$, we use (4.14a) to numerically compute the Hopf bifurcation thresholds $\tau_{H \pm}$ and the imaginary parts $\lambda_{I H \pm}>0$ of the corresponding eigenvalue for both the competition and synchronous modes as a function of $x_{0}$. The results are shown in Fig. 3. Our numerical results strongly suggest that, for either mode of instability and for any $x_{0}>x_{0-}$, there is a unique value of $\tau$ at which there is a Hopf bifurcation. From Fig. 3, we observe that the synchronous mode sets the Hopf bifurcation threshold $\tau_{H m}\left(x_{0}\right)$ for a large range of $x_{0}$, but that there is some range of $x_{0}$ where the Hopf bifurcation threshold $\tau_{H-}$ for the competition threshold is marginally lower than that of the synchronous mode. For $x_{0}$ large, the two thresholds approach the common Hopf bifurcation threshold of a one-spike solution. When $x_{0}>x_{0-}$ and $\tau<\tau_{H m}\left(x_{0}\right)$, the slow repulsive spike dynamics (4.15) is valid, and we obtain

$$
\frac{d x_{0}}{d t}=\frac{\varepsilon_{0}^{2} q}{r-2} \frac{e^{-2 x_{0}}}{1+e^{-2 x_{0}}},
$$

for an initial value satisfying $x_{0}(0)>x_{0-}$.

Generalized GS Model: Next, we consider a generalization of the Gray-Scott model where $p=r=3$, $u_{b}=1, f=-u$, and $g=\mathcal{A} u$ with $\mathcal{A}>0$. For this choice, the nonlinear algebraic equation (2.6) for $U_{0}$ reduces to $\sqrt{U_{0}}\left(1-U_{0}\right)=\left(b_{3} / \mathcal{A}^{3 / 2}\right) G\left(x_{0} ; x_{0}\right)$ where $b_{3} \equiv \int_{-\infty}^{\infty} w^{3} d y$. Since $b_{3}=\sqrt{2} \pi$, we can write the 

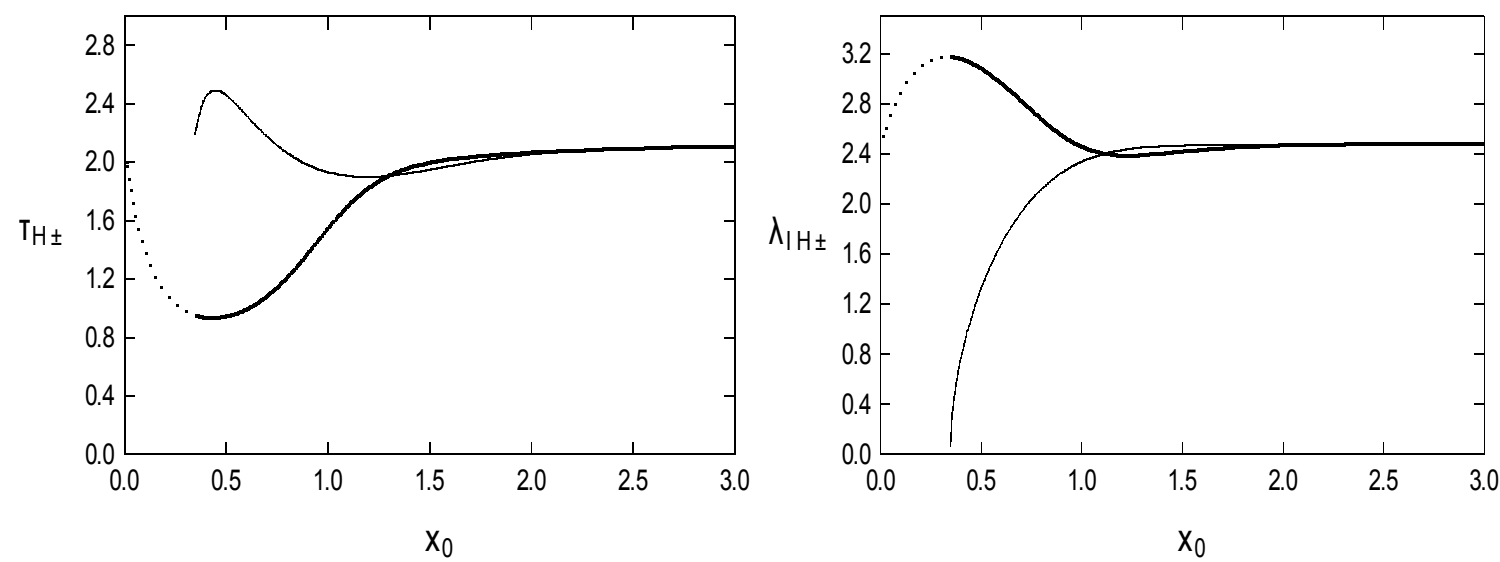

FigURE 3. Hopf bifurcation results for the GM model with $q=2, r=3$, and $s=0$. Left panel: Plot of the Hopf bifurcation thresholds $\tau_{H-}$ and $\tau_{H+}$ for the competition mode (solid curve) and the synchronous mode (heavy solid curve), respectively, as a function of $x_{0}$ on $x_{0}>x_{0-}$. On the range $0<x_{0}<x_{0-}$, where the competition mode is unstable due to a positive real eigenvalue for any $\tau \geq 0$, only the synchronous instability threshold $\tau_{H+}$ exists (dotted curve). Right panel: Plot of the imaginary parts of the corresponding eigenvalue $\lambda_{I H \pm}$ versus $x_{0}$ for both modes, with the same labelling as in the left panel. Observe that $\lambda_{I H_{-}} \rightarrow 0$ as $x_{0} \rightarrow x_{0-}^{+}$.

nonlinear algebraic equation for $U_{0}=U_{0}\left(x_{0}\right)$ as

$$
\mathcal{H}\left(U_{0}\right) \equiv \sqrt{U_{0}}\left(1-U_{0}\right)=\frac{2}{3 \sqrt{3}}\left(\frac{\mathcal{A}_{m}}{\mathcal{A}}\right)^{3 / 2} \quad \text { where } \quad \mathcal{A}=\mathcal{A}_{m}\left(x_{0}\right) \equiv\left[\frac{3 \sqrt{6} \pi}{4}\left(1+e^{-2 x_{0}}\right)\right]^{2 / 3} .
$$

Since $\mathcal{A}>0$, any root to (5.4) must satisfy $0<U_{0}<1$. Moreover, on $0<U_{0}<1$ the function $\mathcal{H}\left(U_{0}\right)$ attains its maximum value of $2 /(3 \sqrt{3})$ at $U_{0}=1 / 3$, with $\mathcal{H}^{\prime}\left(U_{0}\right)>0$ on $0<U_{0}<1 / 3$ and $\mathcal{H}^{\prime}\left(U_{0}\right)<0$ on $1 / 3<U_{0}<1$. Therefore, it follows that the graph of $U_{0}$ versus $\mathcal{A}$ has a saddle-node bifurcation structure, with two solutions $U_{0 \pm}$ for $U_{0}$ in the range $0<U_{0-}<1 / 3<U_{0+}<1$ when $\mathcal{A}>\mathcal{A}_{m}\left(x_{0}\right)$, and no solutions when $\mathcal{A}<\mathcal{A}_{m}\left(x_{0}\right)$.

Since $\mathcal{A}_{m}\left(x_{0}\right)$ is a decreasing function of $x_{0}$, there are three cases that need to be considered in the construction of the two-spike quasi-equilibrium solution.

Case I: $\mathcal{A}<\mathcal{A}_{m}(\infty)$. In this case, $A<\mathcal{A}_{m}\left(x_{0}\right)$ for all $x_{0}>0$, so that (5.4) has no solutions for any $x_{0}>0$. Therefore, on this range of $\mathcal{A}$ there are no symmetric two-spike quasi-equilibria.

Case II: $\mathcal{A}_{m}(\infty)<\mathcal{A}<\mathcal{A}_{m}(0)$. In this case, (5.4) has two solutions only when $x_{0}>x_{0 e}$, where

$$
x_{0 e} \equiv-\frac{1}{2} \log \left(\frac{4}{3 \sqrt{6} \pi} \mathcal{A}^{3 / 2}-1\right)>0 .
$$

Therefore, in this case a two-spike quasi-equilibrium solution exists only when the spikes are sufficiently well-separated. A graphical representation of this case is shown in the left panel of Fig. 4.

Case III: $\mathcal{A}>\mathcal{A}_{m}(0)$. Then, (5.4) has two solutions for all $x_{0}>0$.

In terms of any roots $U_{0 \pm}$ of (5.4), the amplitudes $v_{ \pm}(0)$ of the two possible spikes at $x_{0}$, as obtained from $(2.5)$, are

$$
v_{ \pm}(0)=\frac{w(0)}{\sqrt{\mathcal{A} U_{0 \pm}}}, \quad w(0)=\sqrt{2} .
$$

Therefore, a root $U_{0-}$ on $0<U_{0-}<1 / 3$ generates a "large" amplitude spike, whereas $U_{0+}$ on $1 / 3<$ $U_{0+}<1$ generates a small amplitude spike. 

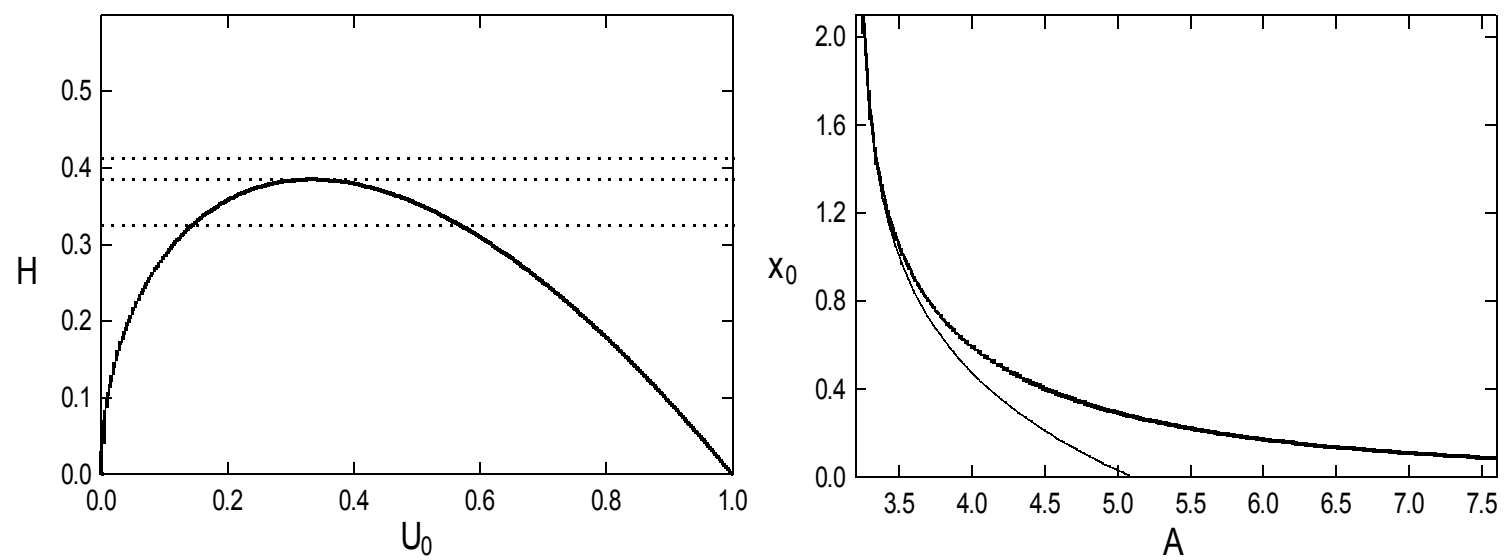

Figure 4. Left panel: Graphical representation of the solution to (5.4) for Case II of the generalized Gray-Scott model where $\mathcal{A}_{m}(\infty)<\mathcal{A}<\mathcal{A}_{m}(0)$ and $\mathcal{A}=4.20$. The heavy solid curve is $\mathcal{H}\left(U_{0}\right)$ while the top, middle, and bottom, dotted horizontal lines are the right-hand side of (5.4) when $x_{0}=0.15, x_{0}=x_{0 e} \approx 0.3553$ and $x_{0}=2$, respectively. Only when $x_{0}>x_{0 e}$ are there two roots to (5.4). Right panel: Plot of the competition instability threshold $x_{0-}$ (heavy solid curve) and the existence threshold $x_{0 e}$ (solid curve) versus $\mathcal{A}$ for a two-spike pattern. Here $x_{0-}$ is the unique root of $\mathcal{B}\left(x_{0}\right)=0$ in (5.8), and $x_{0 e}$ is given in (5.5). When the initial spike location $x_{0}(0)$ satisfies $x_{0}(0)>x_{0}$ and $\tau>0$ is below a Hopf bifurcation threshold, the two-spike quasi-equilibrium solution exhibits slow dynamics.

Next, we will consider the stability properties of the quasi-equilibrium solutions for Case II and Case III. To do so, we use $(4.14 \mathrm{c})$ to calculate $d_{0}, d_{1}$, and $\mathcal{G}(0)$ as

$$
d_{0}=-\frac{2\left(1-U_{0}\right)}{U_{0}\left(1+e^{-2 x_{0}}\right)}<0, \quad d_{1}=-\frac{9\left(1-U_{0}\right)}{U_{0}\left(1+e^{-2 x_{0}}\right)}<0, \quad \mathcal{G}(0)=\frac{\left(1-U_{0}\right)}{U_{0}\left(1+e^{-2 x_{0}}\right)}>0,
$$

where $U_{0}$ is any solution to (5.4).

We first consider the synchronous mode. Suppose that $x_{0}>x_{0 e}$ for Case II and $x_{0}>0$ for Case III. Then, since $d_{0}<0$ and $\mathcal{F}_{+}(0)>0$, Propositions 4.6 and 4.7 prove that the synchronous mode is stable for $\tau=0$ when $\mathcal{F}_{+}(0)<\mathcal{G}(0)$, and is unstable for any $\tau \geq 0$ when $\mathcal{F}_{+}(0)>\mathcal{G}(0)$. Upon using (5.7), we conclude that we have stability for $\tau=0$ when $2<\left(1-U_{0}\right) / U_{0}$ and instability for all $\tau \geq 0$ when $2>\left(1-U_{0}\right) / U_{0}$. Therefore, the synchronous mode is unstable for all $\tau \geq 0$ for the small amplitude spike where $1 / 3<U_{0}<1$, but is stable when $\tau=0$ for the large amplitude spike corresponding to $0<U_{0}<1 / 3$.

Next, we consider the competition mode when $0<U_{0}<1 / 3$. Suppose that $x_{0}>x_{0 e}$ for Case II and $x_{0}>0$ for Case III. From Propositions 4.6 and 4.7, the competition mode is stable for $\tau=0$ when $\mathcal{F}_{-}(0)<\mathcal{G}(0)$ and is unstable for any $\tau \geq 0$ when $\mathcal{F}_{-}(0)>\mathcal{G}(0)$. From (5.7), the condition $\mathcal{F}_{-}(0)<\mathcal{G}(0)$ is satisfied when $\operatorname{coth} x_{0}<\left(1-U_{0}\right) /\left(2 U_{0}\right)$. We conclude that a large amplitude two-spike quasi-equilibrium solution is stable for $\tau=0$ when $\mathcal{B}\left(x_{0}\right)<0$, and is unstable for all $\tau \geq 0$ when $\mathcal{B}\left(x_{0}\right)>0$, where $\mathcal{B}\left(x_{0}\right)$ is defined by

$$
\mathcal{B}\left(x_{0}\right) \equiv \frac{1}{2} \log \left(\kappa\left(U_{0}\right)+1\right)-\frac{1}{2} \log \left(\kappa\left(U_{0}\right)-1\right)-x_{0}, \quad \kappa\left(U_{0}\right) \equiv \frac{\left(1-U_{0}\right)}{2 U_{0}} .
$$

Here $U_{0}=U_{0}\left(x_{0}\right)$ on $0<U_{0}<1 / 3$ satisfies (5.4).

For Case II, we now show that $\mathcal{B}\left(x_{0}\right)=0$ has a unique root on $x_{0 e}<x_{0}<\infty$. To prove this, we note that as $x_{0} \rightarrow x_{0 e}^{+}, U_{0} \rightarrow 1 / 3^{-}$and $\kappa\left(U_{0}\right) \rightarrow 1^{+}$. Thus, $\mathcal{B}\left(x_{0}\right) \rightarrow+\infty$ as $x_{0} \rightarrow x_{0 e}^{+}$. Moreover, as $x_{0} \rightarrow \infty$, 
we have $\mathcal{B}\left(x_{0}\right) \rightarrow-\infty$. Therefore, by the intermediate value theorem there is a root to $\mathcal{B}\left(x_{0}\right)=0$ in $x_{0}>x_{0 e}$. Furthermore, from (5.8) we calculate $\mathcal{B}^{\prime}\left(x_{0}\right)$ as

$$
\mathcal{B}^{\prime}\left(x_{0}\right)=-\frac{2 \kappa^{\prime}\left(U_{0}\right) U_{0}^{\prime}}{\kappa^{2}-1}-1 \text {. }
$$

Since $\kappa^{\prime}\left(U_{0}\right)=-1 /\left(2 U_{0}^{2}\right)<0$ and $U_{0}^{\prime}\left(x_{0}\right)<0$ on $0<U_{0}<1 / 3$, then $\mathcal{B}^{\prime}\left(x_{0}\right)<0$. Thus, $\mathcal{B}\left(x_{0}\right)=0$ has a unique root $x_{0-}$ with $x_{0-}>x_{0 e}$. The proof that there is a unique root $x_{0-}$ for $\mathcal{B}\left(x_{0}\right)=0$ in $x_{0}>0$ for Case III follows from the facts that $\mathcal{B}(0)>0, \mathcal{B}\left(x_{0}\right) \rightarrow-\infty$ as $x_{0} \rightarrow \infty$, and $\mathcal{B}^{\prime}\left(x_{0}\right)<0$ for $x_{0}>0$. In the right panel of Fig. 4 we plot the numerically computed existence threshold $x_{0 e}$ and the competition instability threshold $x_{0-}$ versus $\mathcal{A}$.

In summary, our main conclusions from Propositions 4.6-4.8 are as follows. Case I: There are no two-spike quasi-equilibrium solutions when $\mathcal{A}<\mathcal{A}_{m}(\infty)$. Case II: Let $\mathcal{A}$ satisfy $\mathcal{A}_{m}(\infty)<\mathcal{A}<\mathcal{A}_{m}(0)$. Two different symmetric two-spike quasi-equilibrium solutions exist only when $x_{0}>x_{0 e}$, where $x_{0 e}$ is defined in (5.5). The solution with the small spike amplitude is unstable for all $\tau \geq 0$ to a synchronous instability of the spike amplitude. On the range $x_{0 e}<x_{0}<x_{0-}$, where $x_{0-}$ is the unique root of (5.8), the quasi-equilibrium solution with the large spike amplitude is unstable to a competition instability for all $\tau \geq 0$. On the range $x_{0}>x_{0-}$, the large amplitude two-spike quasi-equilibrium solution is stable when $\tau=0$ and both the competition and synchronous modes undergo a Hopf bifurcation at some $\tau=\tau_{H-}>0$ and $\tau=\tau_{H+}>0$ respectively. For $\mathcal{A}=4.0$, we use (4.14a) to numerically compute $\tau_{H \pm}$ and the imaginary parts $\lambda_{I H \pm}>0$ of the corresponding eigenvalue for both the competition and synchronous modes as a function of $x_{0}$. The numerical results shown in Fig. 5 suggest that, for any $x_{0}>x_{0-}$ and for either mode of instability, there is a unique value of $\tau$ where a Hopf bifurcation occurs. From Fig. 5 , we observe that the synchronous mode sets the Hopf bifurcation threshold $\tau_{H m}\left(x_{0}\right)$ for intermediate values of $x_{0}$ with $x_{0}>x_{0-}$, and that the thresholds for the synchronous and competition modes are essentially indistinguishable for $x_{0}>1.0$. Case III: Let $\mathcal{A}>\mathcal{A}_{m}(0)$. The same qualitative stability results hold as in Case II, provided we simply define $x_{0 e}=0$.

When there are no $\mathcal{O}(1)$ time-scale instabilities, the slow spike dynamics, as obtained from (4.15) and $(5.7)$, is

$$
\frac{d x_{0}}{d t}=\frac{\varepsilon_{0}^{2}}{1+e^{-2 x_{0}}}\left(\frac{1-U_{0}}{U_{0}}\right)
$$

where $U_{0}\left(x_{0}\right)$ on $0<U_{0}<1 / 3$ satisfies (5.4).

Generalized GM Model: Finally, we consider a generalization of the GM model where $p=r=3$, $u_{b}=0, f=1$, and $g=u^{-1}+\alpha$, where $\alpha>0$ is a parameter. This choice for the nonlinearities is closely related to the example in $\S 5.2$ of [5] where the prototypical GM nonlinearities with $p=r=2$ was considered. In [5] the slow spike dynamics was analyzed, but no NLEP stability analysis was undertaken. For our slightly different choice $p=r=3$ of exponents we are able to provide this stability theory.

For this example, the nonlinear algebraic equation (2.6) for $U_{0}=U_{0}\left(x_{0}\right)$ is

$$
\mathcal{C}\left(U_{0}\right) \equiv\left(U_{0}^{-1 / 3}+\alpha U_{0}^{2 / 3}\right)=\left[\sqrt{2} \pi G\left(x_{0} ; x_{0}\right)\right]^{2 / 3}, \quad G\left(x_{0} ; x_{0}\right) \equiv \frac{1}{2}\left(1+e^{-2 x_{0}}\right) .
$$

The function $\mathcal{C}\left(U_{0}\right)$ has a global minimum on $U_{0}>0$ at the point $U_{0 m}=1 /(2 \alpha)$, with $\mathcal{C}^{\prime}\left(U_{0}\right)>0$ for $U_{0}>U_{0 m}$ and $\mathcal{C}^{\prime}\left(U_{0}\right)<0$ for $0<U_{0}<U_{0 m}$. The minimum value is $\mathcal{C}_{\min }=\mathcal{C}\left(U_{0 m}\right)$. Since the right-hand side of $(5.9)$ is a decreasing function of $x_{0}$, there are three cases to be considered.

Case I: $[\sqrt{2} \pi G(0 ; 0)]^{2 / 3}<\mathcal{C}_{\min }$, which holds when $\alpha>\alpha_{\max }$, where

$$
\alpha_{\max }=\frac{2 \pi^{2}}{\left(2^{1 / 3}+2^{-2 / 3}\right)^{3}} .
$$

In this case (5.9) has no solutions for any $x_{0}>0$, and so there are no symmetric two-spike quasi-equilibria. 

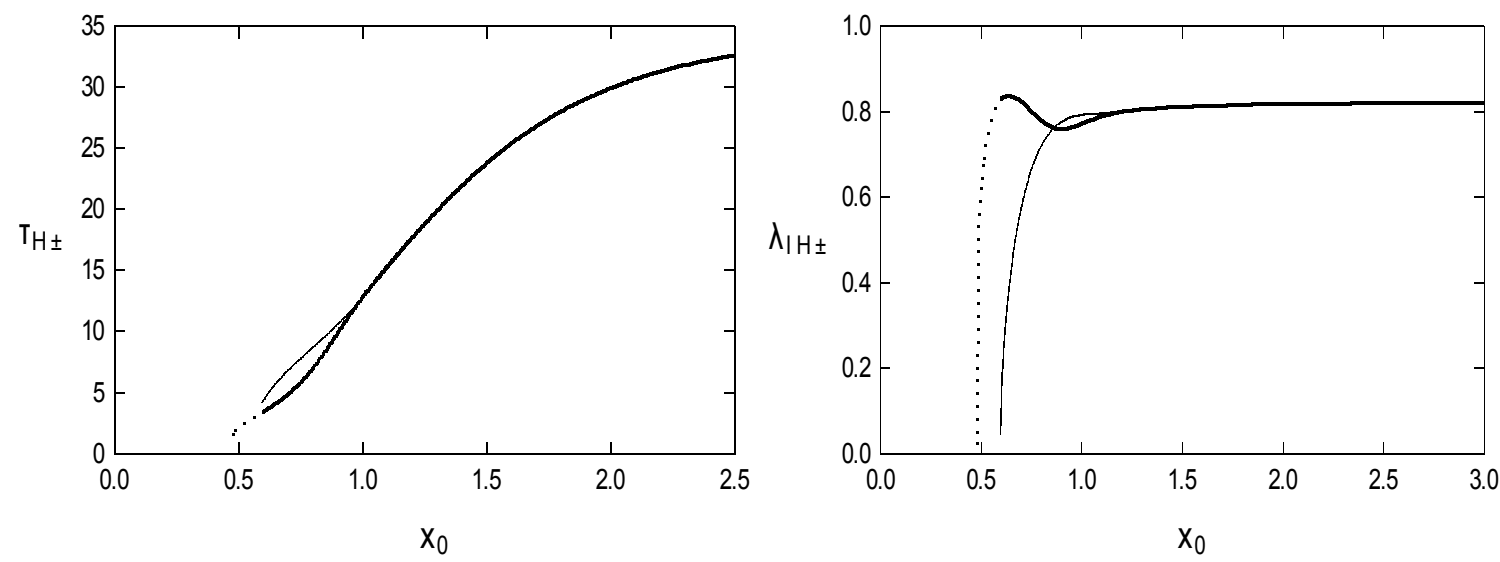

FigURE 5. Hopf bifurcation results for the GS model with $\mathcal{A}=4.0$, which corresponds to Case II since $3.218 \approx \mathcal{A}_{m}(\infty)<\mathcal{A}<\mathcal{A}_{m}(0) \approx 5.101$. Left panel: Plot of the Hopf bifurcation thresholds $\tau_{H-}$ and $\tau_{H+}$ for the competition mode (solid curve) and the synchronous mode (heavy solid curve), respectively, as a function of $x_{0}$ on $x_{0}>$ $x_{0-} \approx 0.5946$. On the range $0.4758 \approx x_{0 e}<x_{0}<x_{0-}$, where the competition mode is unstable due to a positive real eigenvalue for any $\tau \geq 0$, only the synchronous instability threshold $\tau_{H+}$ exists (dotted curve). Right panel: Plot of the imaginary parts of the corresponding eigenvalue $\lambda_{I H \pm}$ versus $x_{0}$ for both modes, with the same labelling as in the left panel. Observe that $\lambda_{I H_{-}} \rightarrow 0$ as $x_{0} \rightarrow x_{0-}^{+}$and $\lambda_{I H+} \rightarrow 0$ as $x_{0} \rightarrow x_{0 e}^{+}$.

Case II: $[\sqrt{2} \pi G(\infty ; \infty)]^{2 / 3}<\mathcal{C}_{\min }<[\sqrt{2} \pi G(0 ; 0)]^{2 / 3}$, which holds for $\alpha$ on the range $\alpha_{\max } / 4<$ $\alpha<\alpha_{\max }$. Then, (5.9) has two solutions $U_{0 \pm}$ with $0<U_{0-}<U_{0 m}$ and $U_{0 m}<U_{0+}<\infty$ only when $x_{0}$ satisfies $0<x_{0}<x_{0 e}$, where

$$
x_{0 e}=-\frac{1}{2} \log \left(\frac{\sqrt{2}}{\pi} \mathcal{C}_{\text {min }}^{3 / 2}-1\right), \quad \mathcal{C}_{\min } \equiv \alpha^{1 / 3}\left(2^{1 / 3}+2^{-2 / 3}\right) .
$$

There are no two-spike quasi-equilibria when $x_{0}>x_{0 e}$, and a one-spike equilibrium solution does not exist. A graphical illustration of this case is shown in Fig. 6. This qualitative behaviour, whereby a two-spike quasi-equilibrium pattern does not exist when the spikes are too far apart, is in direct contrast to Case II for the generalized GS model, considered above, where the two-spike pattern exists only when the spikes are sufficiently well-separated.

Case III: $\mathcal{C}_{\min }<[\sqrt{2} \pi G(\infty ; \infty)]^{2 / 3}$, which holds for $\alpha<\alpha_{\max } / 4$. Then, (5.9) has two solutions $U_{0 \pm}$ with $0<U_{0-}<U_{0 m}$ and $U_{0 m}<U_{0+}<\infty$ for any $x_{0}>0$.

To determine the stability properties of the quasi-equilibrium solutions for Case II and Case III, we first use (4.14c) to calculate $d_{0}, d_{1}$, and $\mathcal{G}(0)$ as

$$
d_{0}=0, \quad d_{1}=-\frac{9}{\left(1+\alpha U_{0}\right)\left(1+e^{-2 x_{0}}\right)}<0, \quad \mathcal{G}(0)=\frac{3}{\left(1+\alpha U_{0}\right)\left(1+e^{-2 x_{0}}\right)}>0,
$$

where $U_{0}$ is any solution to (5.9).

We first consider the synchronous mode on the range $0<x_{0}<x_{0 e}$ for Case II and on $x_{0}>0$ for Case III. Then, since $d_{0}=0$ and $\mathcal{F}_{+}(0)>0$, Propositions 4.6 and 4.7 show that the synchronous mode is stable for $\tau=0$ when $\mathcal{F}_{+}(0)<\mathcal{G}(0)$, and is unstable for any $\tau \geq 0$ when $\mathcal{F}_{+}(0)>\mathcal{G}(0)$. Upon using (5.12), it follows that the two-spike quasi-equilibrium solution is stable for $\tau=0$ when $0<U_{0}<U_{0 m}=1 /(2 \alpha)$ and is unstable for all $\tau \geq 0$ when $U_{0}>U_{0 m}$. 


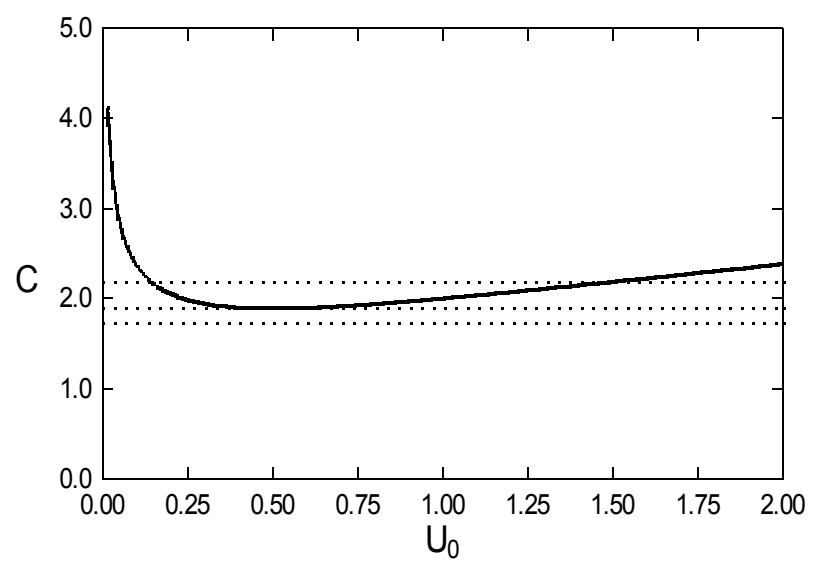

FiguRE 6. Graphical representation of the solution to (5.9) for Case II of the generalized GM model where $\alpha_{\max } / 4<\alpha<\alpha_{\max }$ and $\alpha=1$. The heavy solid curve is $\mathcal{C}\left(U_{0}\right)$ while the top, middle, and bottom, dotted horizontal lines are the right-hand side of (5.9) when $x_{0}=0.40, x_{0}=x_{0 e} \approx 0.887$ and $x_{0}=2$, respectively. Only when $x_{0}<x_{0 e}$ are there two roots to $(5.9)$.

Next, consider the competition mode on the range $0<U_{0}<U_{0 m}$. Suppose that $x_{0}<x_{0 e}$ for Case II and $x_{0}>0$ for Case III. From Propositions 4.6 and 4.7, the competition mode is stable for $\tau=0$ when $\mathcal{F}_{-}(0)<\mathcal{G}(0)$, and is unstable for any $\tau \geq 0$ when $\mathcal{F}_{-}(0)>\mathcal{G}(0)$. From (5.12), the condition $\mathcal{F}_{-}(0)<\mathcal{G}(0)$ is satisfied when $\operatorname{coth} x_{0}<3 /\left[2\left(1+\alpha U_{0}\right)\right]$. This motivates introducing $\mathcal{D}\left(x_{0}\right)$ defined by

$$
\mathcal{D}\left(x_{0}\right) \equiv \frac{1}{2} \log \left(\kappa\left(U_{0}\right)+1\right)-\frac{1}{2} \log \left(\kappa\left(U_{0}\right)-1\right)-x_{0}, \quad \kappa\left(U_{0}\right) \equiv \frac{3}{2\left(1+\alpha U_{0}\right)} .
$$

On the range $0<U_{0}<U_{0 m}$, where $U_{0}\left(x_{0}\right)$ satisfies (5.9), we conclude that a two-spike quasi-equilibrium solution is stable for $\tau=0$ when $\mathcal{D}\left(x_{0}\right)<0$, and is unstable for all $\tau \geq 0$ when $\mathcal{D}\left(x_{0}\right)>0$.

In contrast to the case of the generalized GS model (see equation (5.8)), the function $\mathcal{D}\left(x_{0}\right)$ is not guaranteed to be monotone decreasing. As such, we will consider Case II and Case III separately.

For Case III, where $\alpha<\alpha_{\max } / 4 \approx 0.731$ and a two-spike quasi-equilibrium solution exists for all $x_{0}>0$, it is easily shown that $\mathcal{D}(0)>0$ and $\mathcal{D}\left(x_{0}\right) \rightarrow-\infty$ as $x_{0} \rightarrow+\infty$. Therefore, $\mathcal{D}\left(x_{0}\right)=0$ has a root $x_{0-}>0$. Our numerical results given in the left panel of Fig. 7 show that this root $x_{0-}$ to $\mathcal{D}\left(x_{0}\right)=0$ is unique. Therefore, for $x_{0}>x_{0}$ - and $\tau$ below a Hopf bifurcation threshold, the two-spike quasi-equilibrium exhibits slow dynamics. From (4.15) and (5.12), this slow dynamics is characterized by

$$
\frac{d x_{0}}{d t}=\frac{\varepsilon_{0}^{2}}{\left(1+\alpha U_{0}\right)} \frac{e^{-2 x_{0}}}{\left(1+e^{-2 x_{0}}\right)},
$$

where $U_{0}\left(x_{0}\right)$ on $0<U_{0}<1 /(2 \alpha)$ satisfies (5.9). On the range $0<x_{0}<x_{0 e}$, the two-spike quasiequilibrium solution is unstable for all $\tau \geq 0$.

For Case II, where $0.731 \approx \alpha_{\max } / 4<\alpha<\alpha_{\max } \approx 2.924$, a two-spike quasi-equilibrium solution exists only when $0<x_{0}<x_{0 e}$, where $x_{0 e}$ is given in (5.11). We calculate that $x_{0 e} \rightarrow 0$ as $\alpha \rightarrow 2.924^{-}$, while $x_{0 e} \rightarrow+\infty$ as $\alpha \rightarrow 0.731^{+}$. For this case, we have $\mathcal{D}(0)>0$, but $\mathcal{D}\left(x_{0}\right) \rightarrow+\infty$ as $x_{0} \rightarrow x_{0 e}^{-}$. This latter limit follows from the facts that $U_{0} \rightarrow U_{0 m}^{-}$and $\kappa\left(U_{0}\right) \rightarrow 1^{+}$as $x_{0} \rightarrow x_{0 e}^{-}$. Our numerical computations of the roots of $\mathcal{D}\left(x_{0}\right)=0$ show for $\alpha>0.778$ that $\mathcal{D}\left(x_{0}\right)>0$ for all $x_{0}$ in $0<x_{0}<x_{0 e}$. Therefore, on $0.778<\alpha<2.924$, a two-spike quasi-equilibrium solution is unstable for all $\tau \geq 0$ (see Fig. 7). However, as also shown in Fig. 7, there is a narrow range $0.731<\alpha<0.778$, where $\mathcal{D}\left(x_{0}\right)<0$ on some intermediate range $0<x_{0-l}<x_{0}<x_{0-r}<x_{0 e}$ of $x_{0}$. 

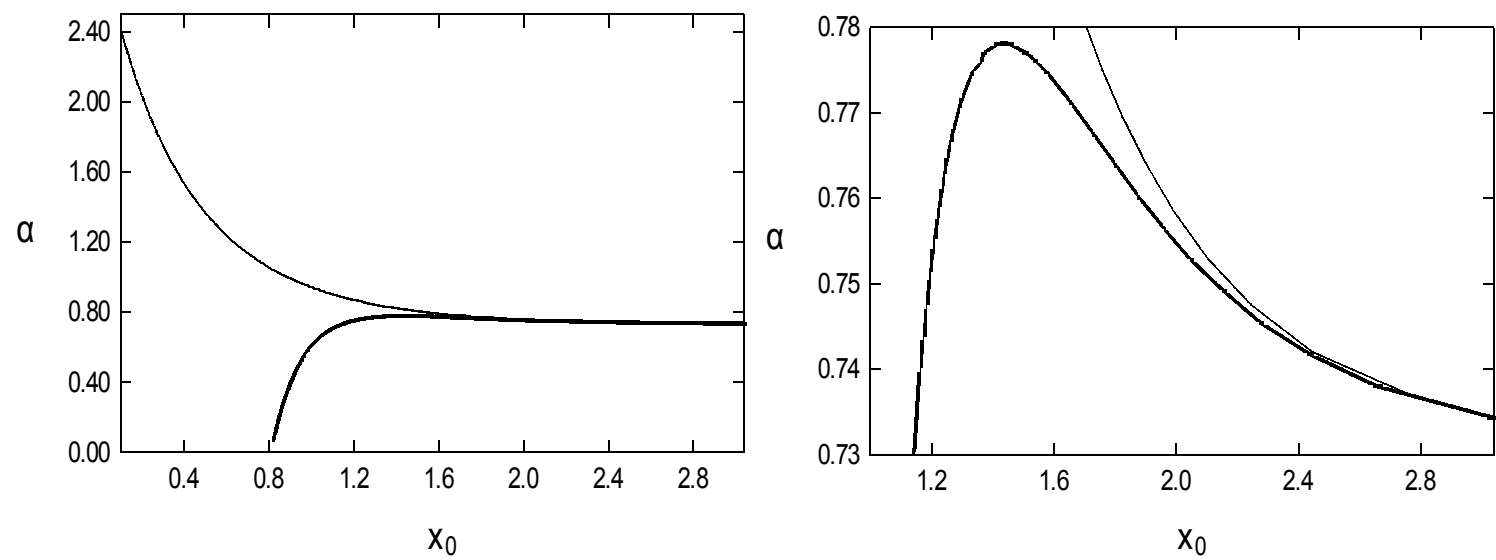

FiguRE 7. Plot of $\alpha$ versus the competition instability threshold $x_{0-}$ (heavy solid curve) and the existence threshold $x_{0 e}$ (solid curve) given in (5.11). The existence threshold is defined only on $0.731=\alpha_{\max } / 4<\alpha<\alpha_{\max } \approx 2.924$. The threshold(s) $x_{0-}$ are the roots of (5.13). For $\alpha<\alpha_{\max } / 4 \approx 0.731, x_{0-}$ is unique and the two-spike pattern is unstable for all $\tau \geq 0$ when $x_{0}<x_{0-}$, and is stable for $\tau=0$ when $x_{0}>x_{0-}$. On the narrow range $0.731=\alpha_{\max } / 4<\alpha<0.778$, there are two roots to (5.13). These roots are shown more clearly in the right panel where we display a zoom of the left panel on this range. Since $\mathcal{D}\left(x_{0}\right)<0$ between these roots, the two-spike pattern is stable for $\tau=0$ on this range of $x_{0}$, and is unstable for any $\tau \geq 0$ for $x_{0}$ outside this range. For $\alpha>0.778$, $\mathcal{D}\left(x_{0}\right)>0$ on $0<x_{0}<x_{0 e}$, and a two-spike quasi-equilibrium solution is unstable for all $\tau \geq 0$.

Therefore, when $0.731<\alpha<0.778$, it is only on this intermediate range of $x_{0}$ that a two-spike quasi-equilibrium solution will exhibit slow spike dynamics for $\tau \geq 0$ sufficiently small. For an initial location $x_{0}(0)=x_{00}$ within this range, the slow dynamics is given by the repulsive interaction (5.14) and, consequently, $x_{0}$ increases slowly in time. However, as $t$ increases, eventually $x_{0}$ exceeds the upper endpoint $x_{0-r}$ of this range, and the two-spike pattern becomes unstable to a fast $\mathcal{O}(1)$ time-scale competition instability. This instability, which results from the slow passage of $x_{0}$ above the stability threshold $x_{0-r}$, corresponds to a dynamically triggered bifurcation event. Our stability theory for Case II, where a one-spike solution does not exist, shows how the two-spike quasi-equilibrium solution loses its stability before $x_{0}$ reaches the existence threshold $x_{0 e}$. The full numerical computations in $\S 5.2$ of [5] of a closely related RD system show that the amplitudes of the two spikes become very large and unequal as $t$ increases, with the RD system eventually exhibiting a finite time blow-up phenomena at later times.

\section{Discussion}

We have characterized the existence, slow dynamics, and linear stability of a symmetric two-spike quasiequilibrium solution to the class of RD systems with regular diffusion (1.1) on the infinite line. A similar analysis has been done for the corresponding sub-diffusive system (1.2). This analysis is an extension of the results obtained in [25] for one-spike solutions. Although the construction of these symmetric twospike quasi-equilibria and their associated slow dynamics was done for arbitrary $p \geq 2$ and $r>1$, for the linear stability theory we considered only the sub-range $p=2 r-3$ and $r>2$ of exponents for which the determination of the discrete spectrum of the associated NLEP can be reduced to the problem of locating the roots of certain relatively simple transcendental equations in the eigenvalue parameter. There are two such transcendental equations, which depend on the two possible modes of instability, consisting of either a synchronous instability or a symmetry-breaking (competition) instability of the spike amplitudes. 
These transcendental equations involve two key parameters $d_{0}$ and $d_{1}$ related to the specific choices of the nonlinearities $f(u)$ and $g(u)$ in (1.1) and (1.2).

There are two further directions that would be of interest to investigate. Firstly, in the context of an explicitly solvable NLEP, it would be interesting to characterize explicitly delayed bifurcation effects on the exchange of stability of localized pulses that results either from the intrinsic slow drift of the pulse locations or from slowly varying extrinsic control parameters, such as $\tau$ or the length of the domain. Although the study of delayed bifurcation effects, and in particular delayed Hopf bifurcations, in the context of ODE models is well-advanced, there are only very limited analytical results of this nature in a genuine PDE context. One main theoretical obstacle for an analysis of delayed bifurcations in PDE's is to be able to locate accurately the spectrum of the linearization for the problem with "frozen" control parameter. In the context of RD systems in the semi-strong limit with an explicitly solvable NLEP, this spectrum is readily available. Secondly, it would be interesting to investigate whether more general, other than power-law, nonlinearities can also lead to an explicitly solvable NLEP's. Results in this direction are given in [32].

Acknowledgements. M. J. W. is grateful for the grant support of NSERC.

\section{References}

[1] W. Chen, M. J. Ward. Oscillatory instabilities of multi-spike patterns for the one-dimensional Gray-Scott model. Europ. J. Appl. Math., 20 (2), (2009), pp. 187-214.

[2] A. Doelman, W. Eckhaus, T. J. Kaper. Slowly modulated two-pulse solutions in the Gray-Scott model $i$ : asymptotic construction and stability. SIAM J. Appl. Math., 1 (3), (2000), pp. 1080-1102.

[3] A. Doelman, W. Eckhaus, T. J. Kaper. Slowly modulated two-pulse solutions in the Gray-Scott model ii: geometric theory, bifurcations, and splitting dynamics. SIAM J. Appl. Math., 61 (6), (2000), pp. 2036-2061.

[4] A. Doelman, R. A. Gardner, T. Kaper. Large stable pulse solutions in reaction-diffusion equations. Indiana U. Math. Journ., 50 (1), (2001), pp. 443-507.

[5] A. Doelman, T. Kaper. Semistrong pulse interactions in a class of coupled reaction-diffusion equations. SIAM J. Appl. Dyn. Sys., 2 (1), (2003), pp. 53-96.

[6] A. Doelman, T. Kaper, K. Promislow, Nonlinear asymptotic stability of the semi-strong pulse dynamics in a regularized Gierer-Meinhardt model. SIAM J. Math. Anal. 38 (6), (2007), pp. 1760-1789.

[7] J. Ehrt, J. D. Rademacher, M. Wolfrum, First and second order semi-strong interaction of pulses in the Schnakenburg model. preprint, (2012).

[8] A. Gierer, H. Meinhardt. A theory of biological pattern formation. Kybernetik, 12, (1972), pp. 30-39.

[9] A. A. Golovin, B. J. Matkowsky, V. A. Volpert, Turing pattern formation in the Brusselator model with superdiffusion. SIAM J. Appl. Math. 69 (1), (2008), pp. 251-272.

[10] P. Gray, S. K. Scott. Autocatalytic reactions in the isothermal, continuous stirred tank reactor: oscillations and instabilities in the system $A+2 B \rightarrow 3 B, B \rightarrow C$. Chem. Eng. Sci. 39, (1984), pp. 1087-1097.

[11] B. I. Henry, S. L. Wearne. Existence of Turing instabilities in a two-species fractional reaction-diffusion system. SIAM J. Appl. Math., 62(3), (2002), pp. 870-887.

[12] D. Iron, M. J. Ward, J. Wei. The stability of spike solutions to the one-dimensional Gierer-Meinhardt model. Physica D, 150 (1-2), (2001), pp. 25-62.

[13] D. Iron, M. J. Ward, The dynamics of multi-spike solutions to the one-dimensional Gierer-Meinhardt model. SIAM J. Appl. Math. 62 (6), (2002), pp. 1924-1951.

[14] T. Kolokolnikov, M. Ward, J. Wei. The stability of spike equilibria in the one-dimensional Gray-Scott model: the low feed-rate regime. Studies in Appl. Math. 115 (1), (2005), pp. 21-71

[15] T. Kolokolnikov, M. Ward, J. Wei. The stability of steady-state hot-spot patterns for a reaction-diffusion model of urban crime. Disc. Cont. Dyn. Sys Series B., to appear, (2013), (34 pages).

[16] C. S. Lin, W. M. Ni, I. Takagi. Large amplitude stationary solutions to a chemotaxis system. J. Diff. Eq. 72 (1), (1988), pp. 1-27.

[17] H. Meinhardt. The Algorithmic Beauty of Sea Shells. Springer-Verlag, Berlin, (1995).

[18] R. Metzler, J. Klafter. The random walk's guide to anomalous diffusion: a fractional dynamics approach. Phys. Rep. 339 (2000), pp. 1-77.

[32] I. Moyls, W. H. Tse, M. J. Ward. On explicitly solvable nonlocal eigenvalue problems and the stability of localized pulses. to be submitted, Applied Math Letters, (2013).

[20] C. Muratov, V. V. Osipov. Stability of the static spike autosolitons in the Gray-Scott model. SIAM J. Appl. Math. 62 (5), (2002), pp. 1463-1487.

[21] Y. Nec, V. A. Volpert, A. A. Nepomnyashchy. Front propagation problems with sub-diffusion. Discr. Cont. Dyn. Sys. Series A. 27 (2), (2010), pp. 827-846. 
[22] Y. Nec, A. A. Nepomnyashchy. Linear stability of fractional reaction-diffusion systems. Math. Model. Nat. Phenom. 2 (2), (2007), pp. 77-105.

[23] Y. Nec, A. A. Nepomnyashchy. Turing instability in sub-diffusive reaction-diffusion systems. J. Physics A: Math. Theor. 40 (49), (2007), pp. 14687-14702.

[24] Y. Nec, M. J. Ward. The dynamics and stability of spike-type solutions to the Gierer-Meinhardt model with subdiffusion. Physica D. 241 (10), (2012), pp. 947-963.

[25] Y. Nec, M. J. Ward, An explicitly solvable nonlocal eigenvalue problem and the stability of a spike for a class of reaction-diffusion systems. Math. Model. of Nat. Phenom. 8 (2), (2013), pp. 55-87.

[26] Y. Nec Spike-type solutions to the one-dimensional Gierer-Meinhardt model with Lévy flights. Studies Appl. Math. 129 (3), (2012), pp. 272-299

[27] K. B. Oldham, J. Spanier. The fractional calculus. Academic Press, New York, 1974.

[28] I. Podlubny. Fractional differential equations. Academic Press, San Diego, 1999.

[29] J. D. Rademacher, First and second order semi-strong interface interaction in reaction-diffusion systems. SIAM J. App. Dyn. Syst. 12 (1), (2013), pp. 175-203.

[30] R. K. Saxena, A. M. Mathai, H. J. Haubold, Fractional reaction-diffusion equations. Astrophys. Space Sci. 305 (3), (2006), pp. 289-296.

[31] W. Sun, M. J. Ward, R. Russell. The slow dynamics of two-spike solutions for the Gray-Scott and Gierer-Meinhardt systems: competition and oscillatory instabilities. SIAM J. App. Dyn. Sys. 4 (4), (2005), pp. 904-953.

[32] I. Moyls, W. H. Tse, M. J. Ward. On explicitly solvable nonlocal eigenvalue problems and the stability of localized pulses. to be submitted, Applied Math Letters, (2013).

[33] A. Turing. The chemical basis of morphogenesis. Phil. Trans. Roy. Soc. B. 327 (1952), pp. 37-72.

[34] J. C. Tzou, A. Bayliss, B. J. Matkowsky, V. A. Volpert, Interaction of Turing and Hopf models in the superdiffusive Brusselator model near a codimension two bifurcation point. Math. Model. Nat. Phenom. 6 (1), (2011), pp. 87-118.

[35] J. C. Tzou, A. Bayliss, B. J. Matkowsky, V. A. Volpert, Stationary and slowly moving localized pulses in a singularly perturbed Brusselator model. Europ. J. Appl. Math. 22 (5), (2011), pp. 423-453.

[36] J. C. Tzou, Y. Nec, M. J. Ward, The Stability of Localized Spikes for the 1-D Brusselator Reaction-Diffusion Model. Europ. J. Appl. Math. 24 (4), (2013), pp. 515-564.

[37] H. van der Ploeg, A. Doelman. Stability of spatially periodic pulse patterns in a class of singularly perturbed reactiondiffusion equations. Indiana U. Math. J. 54 (5), (2005), pp. 1219-1301.

[38] M. J. Ward, J. Wei. Hopf bifurcations and oscillatory instabilities of spike solutions for the one-dimensional GiererMeinhardt model. J. Nonlinear Science, 3 (2), (2003), pp. 209-264.

[39] J. Wei. On single interior spike solutions for the Gierer-Meinhardt system: uniqueness and stability estimates. Europ. J. Appl. Math. 10 (4), (1999), pp. 353-378.

[40] J. Wei. Existence and stability of spikes for the Gierer-Meinhardt system. book chapter in Handbook of Differential Equations, Stationary Partial Differential Equations. Vol. 5 (M. Chipot ed.), Elsevier, (2008), pp. 489-581.

[41] M. Wolfrum, J. Ehrt. Slow motion of quasi-stationary multi-pulse solutions by semistrong interaction in reactiondiffusion systems. WIAS Preprint 1233 (2007). 